\title{
Taxonomic revision of the South American subgenus Canthon (Peltecanthon) Pereira, 1953 (Coleoptera: Scarabaeidae: Scarabaeinae: Deltochilini)
}

\author{
Luis Gabriel de O.A. NUNES ${ }^{1, *}$, Rafael V. NUNES ${ }^{2}$ \& Fernando Z. VAZ-DE-MELLO ${ }^{3}$ \\ ${ }^{1,2}$ Universidade Federal de Mato Grosso, Instituto de Biociências, Programa de Pós Graduação em \\ Ecologia e Conservação da Biodiversidade, Av. Fernando Correa da Costa, 2367, \\ Boa Esperança, Cuiabá-MT, 78060-900, Brazil. \\ ${ }^{3}$ Universidade Federal de Mato Grosso, Instituto de Biociências, Departamento de Biologia e \\ Zoologia, Av. Fernando Correa da Costa, 2367, Boa Esperança, Cuiabá-MT, 78060-900, Brazil. \\ *Corresponding author: luis.gabriel.bio@gmail.com \\ ${ }^{2}$ Email: rafavnunes@gmail.com \\ 32Email: vazdemello@gmail.com \\ ${ }^{1}$ urn:Isid:zoobank.org:author:BBCA47BF-00F1-4967-8153-DC91FAE5A0E5 \\ ${ }^{2}$ urn:1sid:zoobank.org:author:9ED1789C-F1B2-499F-87B2-87B70FA9A2F3 \\ ${ }^{3}$ urn:1sid:zoobank.org:author:2FF2B7D6-1A6B-43C1-9966-A1A949FB2B05
}

\begin{abstract}
Canthon (Peltecanthon) is revised in this work. The subgenus now includes four species: C. (P.) staigi (Pereira, 1953), C. (P.) haroldi nom. nov., C. (P.) splendidus Schmidt, 1922 and $C$. (P.) terciae sp. nov. All species occur in the Atlantic Forest. Maps, natural history information, specimen data and illustrations are provided.
\end{abstract}

Keywords. Dung beetles, Atlantic Forest, new species, rollers, Canthon staigi, Canthon splendidus, Canthon sulcatus.

Nunes L.G. de O.A., Nunes R.V. \& Vaz-de-Mello F.Z. 2020. Taxonomic revision of the South American subgenus Canthon (Peltecanthon) Pereira, 1953 (Coleoptera: Scarabaeidae: Scarabaeinae: Deltochilini). European Journal of Taxonomy 594: 1-27. https://doi.org/10.5852/ejt.2020.594

\section{Introduction}

There are nine subgenera described in the genus Canthon Hoffmannsegg, 1817: Canthon s. str. (48 species); Glaphyrocanthon Martínez, 1948 (42 spp.); Peltecanthon Pereira, 1953 (3 spp.), Pseudepilissus Martínez, 1954 (14 spp.); Goniocanthon Pereira \& Martínez, 1956 (3 spp.); Nesocanthon Pereira \& Martínez, 1956 (3 spp.); Boreocanthon Halffter, 1958 (15 spp.); Francmonrosia Pereira \& Martínez, 1959 (6 spp.); and Trichocanthon Pereira \& Martínez, 1959 (1 sp.). Beside these, 22 species do not have defined subgenera and are considered incertae sedis in the genus (Halffter \& Martínez 1977; Cupello \& Vaz-de-Mello 2018). 
The subgenus Peltecanthon was first described as a full genus and was later reduced to a subgenus of Canthon by Halffter \& Martínez (1977). It included the following three described species: Canthon staigi (Pereira, 1953), which is the type species of the subgenus; the so-called 'Canthon sulcatus Perty, 1830', which was placed in the subgenus by Pereira (1953); and Canthon splendidus Schmidt, 1922, which was transferred under the erroneous name of 'Canthon auricollis' by Halffter \& Martínez (1977) and later corrected by Vaz-de-Mello \& Cupello (2018a).

The last revisions of Peltecanthon were published in the 1970s. Since then, important updates on the morphological delimitation and distribution of the species of Peltecanthon have provided new insights into the taxonomy of this subgenus (e.g., Ferreira-Neto et al. 2017). Currently, it is necessary to establish in an objective and comparative way the characters that differentiate these species, as well as to update the information about their distributions and to correct possible nomenclatural misunderstandings. With access to a new range of material related to these species, our objective was to conduct a taxonomic review of the subgenus Peltecanthon.

\section{Material and methods}

A total of 1050 specimens from the following institutions and collections were examined (curator in parenthesis):

CEAH = Coleção Entomológica Adolph Hempel, Instituto Biológico, São Paulo, state of São Paulo, Brazil (Sérgio Ide)

CEMT = Setor de Entomologia da Coleção Zoológica da Universidade Federal de Mato Grosso, Departamento de Biologia e Zoologia, Cuiabá, Mato Grosso, Brazil (Fernando Z. Vaz-deMello)

MNHN = Muséum national d'histoire naturelle, Paris, France (Olivier Montreuil and Antoine Mantilleri)

MZSP = Museu de Zoologia, Universidade de São Paulo, São Paulo, São Paulo, Brazil (Carlos Campaner and Sônia Casari)

NHMUK $=$ The Natural History Museum, London, United Kingdom (Max Barclay). The code "BMNH" is used on the specimen labels

NMW = Naturhistorisches Museum Wien, Vienna, Austria (Harald Schillhammer)

SMTD = Senckenberg Naturhistorische Sammlungen, Dresden, Germany (Klaus-Dieter Klass and Olaf Jaeger)

ZSM = Zoologische Staatssammlung München, Munich, Germany (Lars Hendrich, Michael Balke and Katja Neven)

Lists of examined material were separated into type material and non type material. For type material, labels are transcribed ipsis litteris (text between double quotation marks) and are separated by '/, with species names in italics. After the information from each type label, label colour or main characteristics are briefly explained in parentheses. In non type material lists, information from labels was organized as follows: country name, in capital letters (e.g., BRAZIL); department or state name in bold letters (e.g., Rio de Janeiro); the number of males ( $\widehat{\partial}$ or $\partial^{\lambda}$ ) and females ( $q$ or $q \rho$ ); municipality and/or locality (when available); coordinates; date (e.g., 3 Mar. 1986); collector (leg.); collection techniques and environmental/ecological information (when available, collection techniques or other details were transcribed in the original language found on the label; text between double quotation marks); and the repository (e.g., CEMT means deposited in CEMT collection).

Morphological analysis and comparisons were conducted using a Leica stereo microscope model EZ 4. The programs Photo Filter and Combine ZP were used for editing of the images. Maps were constructed 
using Diva Gis, ver. 7.5, while the endophallus structure was drawn using a stereo microscope with a Nikon SMZ 800 camera lucida.

The extraction, treatment and preservation of internal sac sclerites follows the procedure described by Zunino (1978). Internal sac sclerite nomenclature followed Tarasov \& Solodovnikov (2011). External morphology terminology was based on recently published taxonomic and phylogenetic works dealing with the tribe Deltochilini and/or Canthon (Vaz-de-Mello et al. 2011; Tarasov \& Génier 2015).

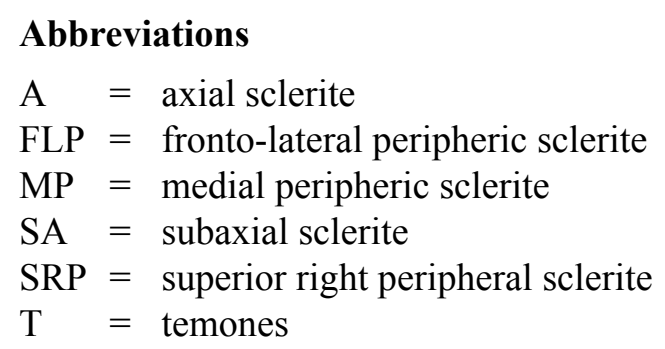

\section{Results}

Class Hexapoda Blainville, 1816

Order Coleoptera Linnaeus, 1758

Family Scarabaeidae Latreille, 1802

Subfamily Scarabaeinae Latreille, 1802

Tribe Deltochilini Lacordaire, 1856

Peltecanthon Pereira, 1953

Peltecanthon Pereira, 1953: 396.

Peltecanthon - Pereira \& Martínez 1956: 94, 98, 182 - Martínez 1959: 26 - Halffter 1961: 230 Vulcano \& Pereira 1964: 573 - Halffter \& Martínez 1967: 80.

Canthon (Peltecanthon) - Halffter \& Martínez 1977: 75 - Vaz-de-Mello 2000: 191 (species list) Medina et al. 2003: 24, 38, 45 - Vaz-de-Mello et al. 2011: 52 (key) - Cupello \& Vaz-de-Mello 2018: 18, 46 - Vaz-de-Mello \& Cupello 2018a: 46.

\section{Type-species}

Peltecanthon staigi Pereira 1953, by original designation (Pereira 1953).

\section{Diagnosis}

Among the Scarabaeinae, the tribe Deltochilini features the following characters: hind wing vein radio posterior 1 (RP1) with a small basal appendix and radio anterior 4 (RA4) clearly thinner than RP1 and basally fused with RP1 (Tarasov \& Dimitrov 2016). Among the Deltochilini, Canthon (Peltecanthon) can be differentiated by the following combination of characters: shiny pronotum and elytral disc, without tubercles or lateral carinae; ventral surface of the metafemora without anterior margin; and metafemora with elongated base, giving the femur a claviform shape (Halffter \& Martínez 1977; Vazde-Mello et al. 2011). 


\section{Key to the species of Canthon (Peltecanthon)}

1. Dorsal surface with blue, green and red metallic reflections (Figs 1A, 3A, 5A, 7A). Metafemur base narrow, claviform, ventral surface without anterior margin (Figs 1B, 3B, 5B, 7B)

.2 Subgenus Peltecanthon Pereira, 1953

- Dorsal surface having other colours and reflections. Metafemur base wide, not claviform, ventral surface with or without anterior margin other Canthon groups and subgenera

2. Dorsal surface with green metallic reflections (Fig. 3A). Metafemora with posterior longitudinal groove at their basal third (Fig. 3B). Hypomera lacking transverse carina (Fig. 3C). Pygidium basal margin (which separates it from pro-pygidium) lacking carina (Fig. 3D). Paramere apical margin sinuous (Fig. 4A). Brazilian Atlantic Forest (from Bahia to Rio de Janeiro)

Canthon (Peltecanthon) haroldi nom. nov.

- Dorsal surface with blue, green and/or red reflections (Figs 1A, 5A, 7A). Metafemur base is smooth (Fig. 7A) or with punctures (Figs 1A, 5A), always lacking longitudinal groove. Hypomera with transverse carina (Figs 1C, 5C, 7C). Pygidium basal margin (which separates it from pro-pygidium) carinate (with a V-shaped projection) (Figs 1D, 5D, 7D). Paramere apical margin straight, substraight or sinuous

3. Elytra and pronotum of different colours; dorsal surface of elytra blue, pronotum with green and red reflections (Fig. 5A). Hypomera with transverse carina strongly marked and interrupted near external margin (Fig. 5C). Pygidium basal margin with weak carina (Fig. 5D). Paramere apical margin strongly sinuous (Fig. 6A). Southern Brazilian Atlantic Forest (Minas Gerais, Espírito Santo and Rio de Janeiro .............................................eanthon (Peltecanthon) splendidus Schmidt, 1922

- Elytra and pronotum of equal colours; dorsal surface of elytra and pronotum blue, red or green. Hypomera with transverse carina finely marked and interrupted at mid-portion (e.g., Figs 1C, 7C). Pygidium basal margin with strong carina. Paramere apical margin sub-straight (e.g., Figs 2A, 8A) .

4. Dorsal surface with blue or green reflections, pronotum and elytra of same colour (Fig. 1A). Metafemur with basal portion punctures (Fig. 1B). Atlantic Forest in Brazil (from Pernambuco to Paraná), Paraguay and Argentina Canthon (Peltecanthon) staigi (Pereira, 1953)

- Dorsal surface with red or light green reflections, pronotum and elytra of same colour (Fig. 7A). Metafemur with basal portion smooth, lacking punctures (Fig. 7A). Northern Brazilian Atlantic Forest (Rio Grande do Norte, Paraíba, Pernambuco and Sergipe)

Canthon (Peltecanthon) terciae sp. nov.

\section{Canthon (Peltecanthon) staigi (Pereira, 1953)}

Figs 1-2

Peltecanthon staigi Pereira, 1953: 396, 399, figs 2-12 (description).

Canthon smaragdulus [error] - Costa Lima 1953: 23, fig. 12 (catalogue).

Peltecanthon staigi - Pereira \& Martínez 1956: 98 (species list) - Martínez 1959: 26 (catalogue) - Vulcano \& Pereira 1964: 573 (catalogue) - Halffter \& Martínez 1967: 80 (monograph) Cupello \& Vaz-de-Mello 2018: 18.

Canton (Peltecanthon) staigi - Halffter \& Martínez 1977: 75 (taxonomic remarks) -Medina et al. 2003: 29-30, 34-35, 38-39, 42-43, 45-47, 50, 52, 54, 57, 64, 66 - Vaz-de-Mello et al. 2011: 52, fig. 31 (key) - Cupello \& Vaz-de-Mello 2018: 46 - Vaz-de-Mello \& Cupello 2018a: 46. 


\section{Diagnosis}

In the subgenus, Canthon staigi can be distinguished by the following combination of characters. Dorsally elytra and pronotum with blue or green colouration and blue and green reflections (Fig. 1A). Metafemora with several punctures at base, with no groove (Fig. 1B). Hypomere with thin transverse carina between the anterior and posterior portions, projecting to the median region, anterior region not excavated (Fig. 1C). Propygidium with strong V-shaped carina between pygidium and propygidium (Fig. 1D). Parameres flattened in lateral view (Fig. 2A); truncated apex, sub-straight, never sinuous; slightly rounded upper angle; straight lower angle. In dorsal view (Fig. 2B): membranous portion separates the parameres, wider in the central region and narrower at the base and apex. Internal sac (Fig. 2C-D) with peripheral fronto-lateral sclerite (FLP) involving axial sclerite (A), both structures forming a complex with a central duct, wider at the base, gradually narrowing toward the apex, which is much finer; subaxial sclerite (SA) lateral to the FLP + A complex; peripheral medial sclerite (MP) lateral in relation to FLP + A, this with the sharper part passing within a semiduct formed by MP; MP with a central denticle at the inner margin (Fig. 2C); superior right peripheral sclerite (SRP) circular and with semi-circular lateral prolongation.

\section{Material examined}

\section{Holotype}

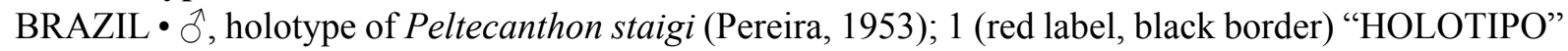
/ 2 (white label, black margins) "Espirito Santo, Col. Mutum, 16849" / 3 (white label, black margins) "Canthon smaragdulus $\widehat{~}$, Lüd. det. 14" / 4 (white label, black margins) "Peltecanthon staigi, gen.

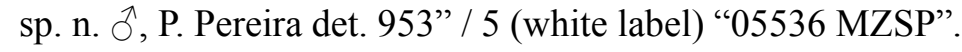

Other material (630 specs)

BRAZIL - Alagoas • 2 ठð $\partial^{\lambda}$; Ibateguara, Coimbra; 10 Nov. 2007; B.K.C. Figueiras leg.; "fezes”; CEMT

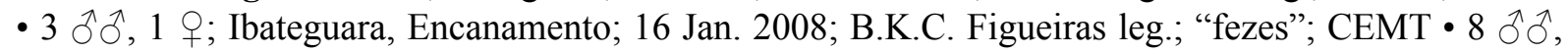
2 우; same collection data as for preceding; "carcaça"; CEMT $\bullet 2 \widehat{\jmath}, 1$ + ; Ibateguara, Cachoeira; 20 Feb. 2008; B.K.C. Figueiras leg.; "fezes”; CEMT • 1 क; União dos Palmares; Jun. 1998; CEMT • 1 đ’; Rio Largo; 7 May 2018; Pedro leg; CEMT. - Pernambuco • 1 đ; Caruaru, Parque Ecológico João Vasconcelos Sobrinho; 6 Nov. 2007; D. Cavalcante et al. leg.; "Mata Atlântica"; pitfall TD/2F 22Z;

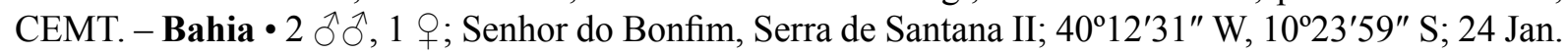
2006; P.P. Lopes leg.; "isca indet." (F/C); CEMT • 1 đ, 1 \&; Lençois, 4 km NW de Lençois e Pai Inácio;

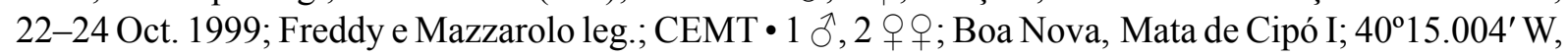

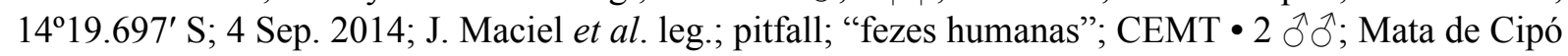
II; $40^{\circ} 12.368^{\prime} \mathrm{W}, 1^{\circ} 21.006^{\prime} \mathrm{S}$; 4 Sep. 2014; J. Maciel et al. leg.; pitfall; "fezes humanas"; CEMT •

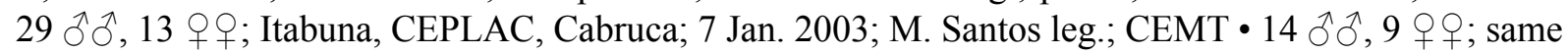
collection data as for preceding; $10 \mathrm{Jan} .2003$; CEMT • $2 \mathrm{o}^{\lambda} \mathrm{o}^{\prime}, 2$ 우 ; Itapetinga; $39^{\circ} 57^{\prime} 54^{\prime \prime} \mathrm{W}, 15^{\circ} 15^{\prime} 50^{\prime \prime} \mathrm{S}$; 280 m a.s.1.; Dec. 2011; Parc62; pitfall; C.M.P. Leite leg., CEMT $\bullet 1$ क; same collection data as for

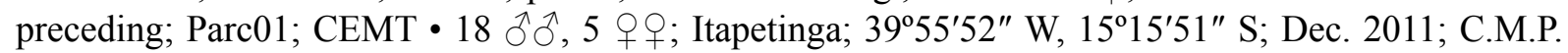

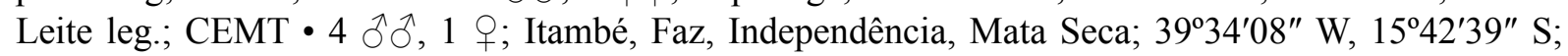

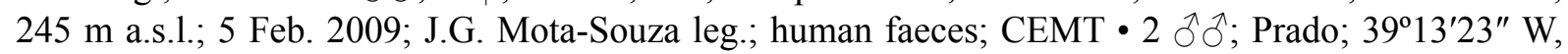

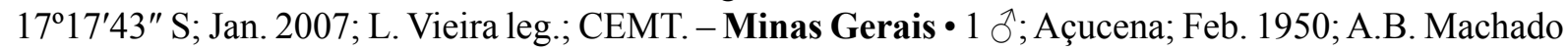
leg.; CEMT • 1 \&; same collection data as for preceding; Feb. 1952; CEMT • 3 q $\odot$; Ipatinga; Nov. 1991; E. Grossi leg.; CEMT • 1 ऊं; same collection data as for preceding; Dec. 1987; CEMT • 1 O ; Marliéria, Parque Estadual do Rio Doce; 4132' W, 19³9' S; 12 Jun. 1994; M.F. Vasconcelos leg.; "mata"; "coletado em fezes na trilha do vinhático"; $n^{\circ}$ de Registro M.F. Vasconcelos 031; CEMT • 1 spec.; Matipeo; Jul.

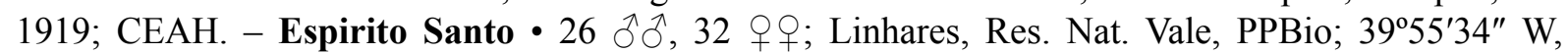

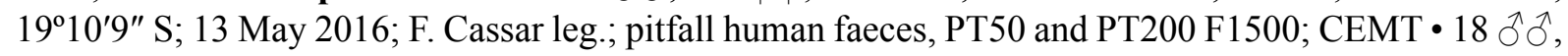
12 우; same collection data as for preceding; $39^{\circ} 56^{\prime} 8^{\prime \prime} \mathrm{W}, 19^{\circ} 10^{\prime} 10^{\prime \prime} \mathrm{S}$; pitfall human faeces, PT0 


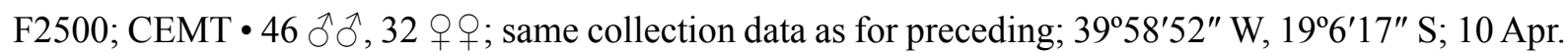
2016; pitfall human faeces, PT50, PT100, PT150, PT200 and PT250 I500; CEMT • 39 ô ô, 50 우우; same collection data as for preceding; $40^{\circ} 0^{\prime} 27^{\prime \prime} \mathrm{W}, 1^{\circ} 8^{\prime} 44^{\prime \prime} \mathrm{S}$; 16 Apr. 2016; pitfall human faeces, PT0,

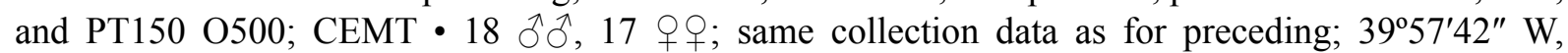

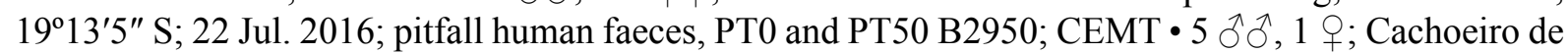
Itapemirim; 5 Jan. 1991; J.C.N. Louzada leg.; CEMT • 1 đ, 1 q; Cachoeiro de Itapemirim, Itabira; 8 Jul. 1995; J.C.N. Louzada leg.: CEMT - 1 क; Linhares, Reserva Natural Vale, PPBio; 3957'52" W, $19^{\circ} 06^{\prime} 18^{\prime \prime}$ S; 25 Jul. 2016; F. Cassar leg.; pitfall human faeces; CEMT • 1 \%; same collection data as for preceding; 39 58 $27^{\prime \prime} \mathrm{W}, 1^{\circ} 06^{\prime} 18^{\prime \prime} \mathrm{S}$; 10 Apr. 2016; CEMT • $1 \mathrm{\delta}^{\Uparrow}$; same collection data as for preceding;

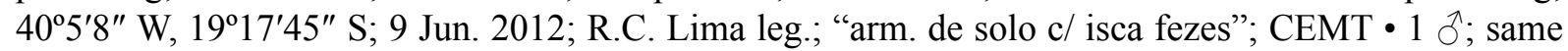

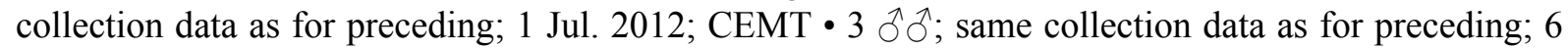

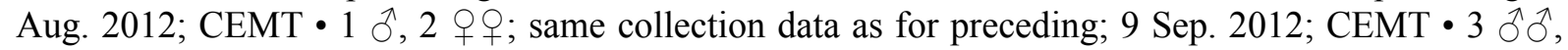
2 우; same collection data as for preceding; 14 Oct. 2012; CEMT • 1 spec.; Linhares, Fazenda Caliman; 23-31 Jan. 2000; S. Ide leg.; CEAH • 1 क; Fazenda, Lagoa do Macacu; 3958'43" W, 19³'50" S; 10 m a.s.1.; 25 Jan. 2000; F. Génier and S. Ide leg.; prim. lowland atlantic for.; sandy, ex. dung tp 1, day 1, FG2000-25; CEMT • 1 O; Regência, Reserva Biológica de Comboios; 3953'5" W, 19³9'57" S; Jan. 2007; L. Vieira leg.; CEMT • $3 \widehat{\partial} \widehat{\partial}$; Matataízes; Jan. 2007; L Vieira leg.; CEMT • 26 $\widehat{\partial}$; 8 우우

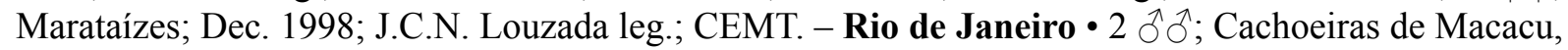
Bacia Guapi-Macacu 4250'6.99" W, 22031'21.72"; Apr. 2016; P.D. Tavares and T. Carvalho leg.; "isca

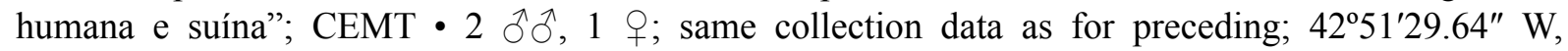

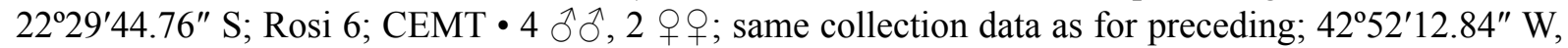

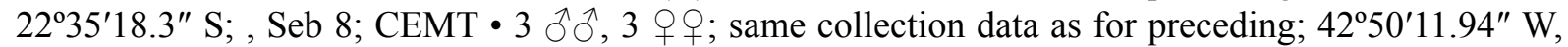

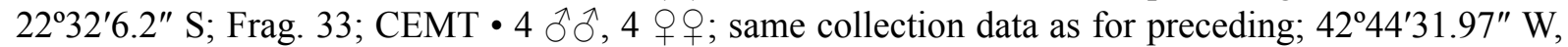
$22^{\circ} 32^{\prime} 14.81^{\prime \prime}$ S; Frag. 42 e 43; CEMT • 2 specs; Oct. 1928; CEAH • 2 ô; Floresta da Tijuca; Nov.

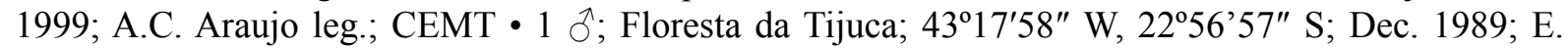

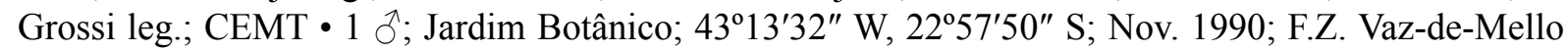

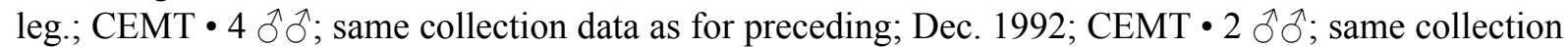

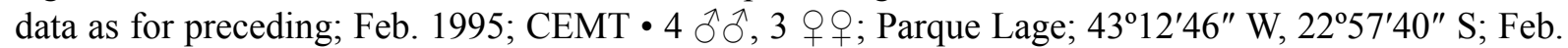
1992; F.Z. Vaz-de-Mello leg.; CEMT • 1 đ; same collection data as for preceding; 11 Sep. 1990; Colibella leg.; CEMT • 1 O ; Jacarepaguá; 4324'59" W, 2256'58" S; Nov. 1990; F.Z. Vaz-de-Mello leg.; CEMT •

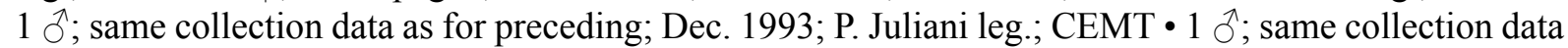

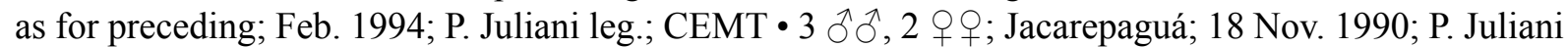

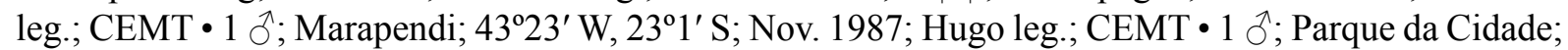

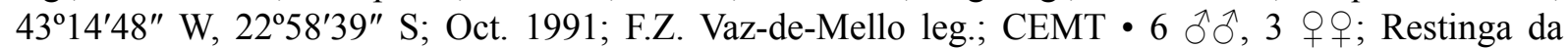

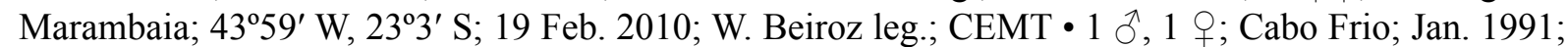

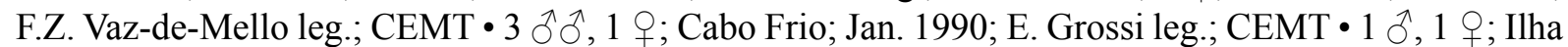
de Cabo Frio; 27 Jan. 1984; Carvalho, Sampaio and Kosawa leg.; CEMT • 1 ภ, 1 क; Itaguai; Jan.

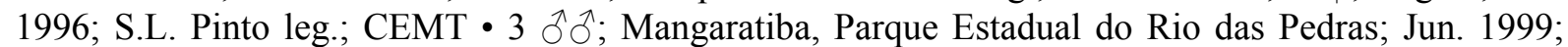
D.M. Takiya leg.; CEMT • 1 §; Miguel Pereira; Dec. 1995; J. Carlos leg.; CEMT • 1 \%; same collection data as for preceding; Mar. 2001; CEMT 1 §; same collection data as for preceding; Dec. 2009; CEMT

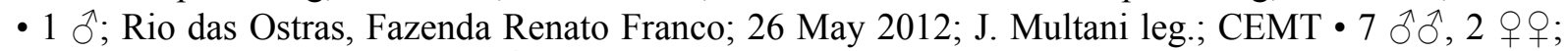
Guapimirim, PARNA Serra dos Órgãos; 22 ${ }^{\circ} 31^{\prime} 27^{\prime \prime}$ S, $43^{\circ} 0^{\prime} 2^{\prime \prime}$ W; 150 m a.s.1.; 18 Dec. 2014; C. Araújo

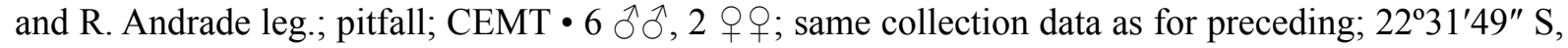
$43^{\circ} 0^{\prime} 4^{\prime \prime} \mathrm{W} ; 100 \mathrm{~m}$ a.s.1.; CEMT • 1 क; same collection data as for preceding; $22^{\circ} 29^{\prime} 41^{\prime \prime} \mathrm{S}, 43^{\circ} 0^{\prime} 8^{\prime \prime} \mathrm{W}$; 400 m a.s.l.; CEMT • 1 O, 3 우; [Mangaratiba], Itacuraça [Itacuruça]; 20 Jul.1982; CEMT. - São Paulo

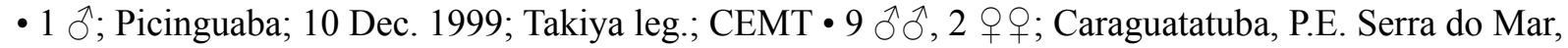

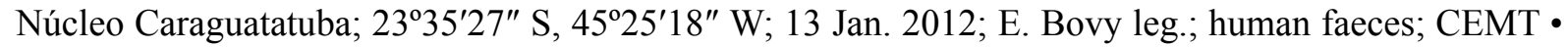

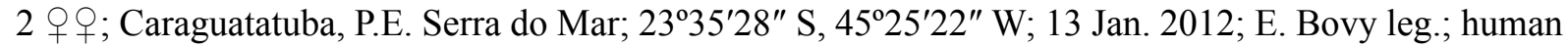

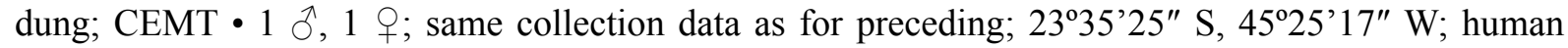

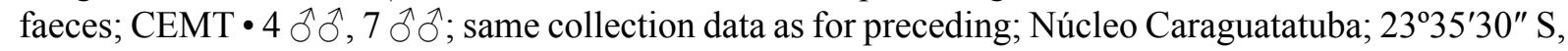



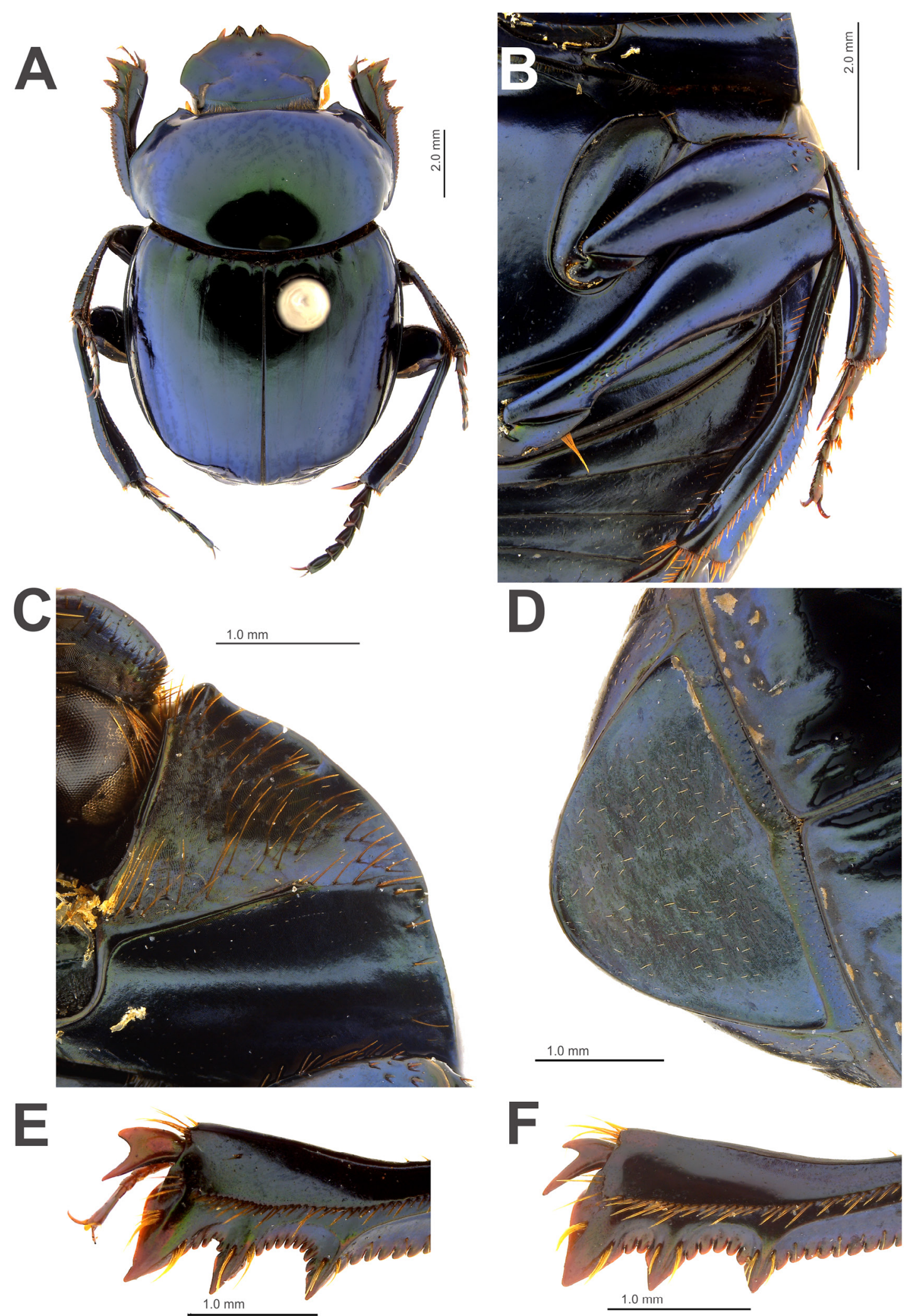

Fig. 1. Canthon (Peltecanthon) staigi (Pereira, 1953), Ô, São Paulo, Bertioga (CEMT). A. Dorsal habitus. B. Ventral surface of meso- and metafemora. C. Transverse carina in the hypomeron. D. Carina in the margin between pygidium and propygidium. E. Male protibial spur. F. Female protibial spur, São Paulo, Caraguatatuba, P.E. Serra do Mar (CEMT). 

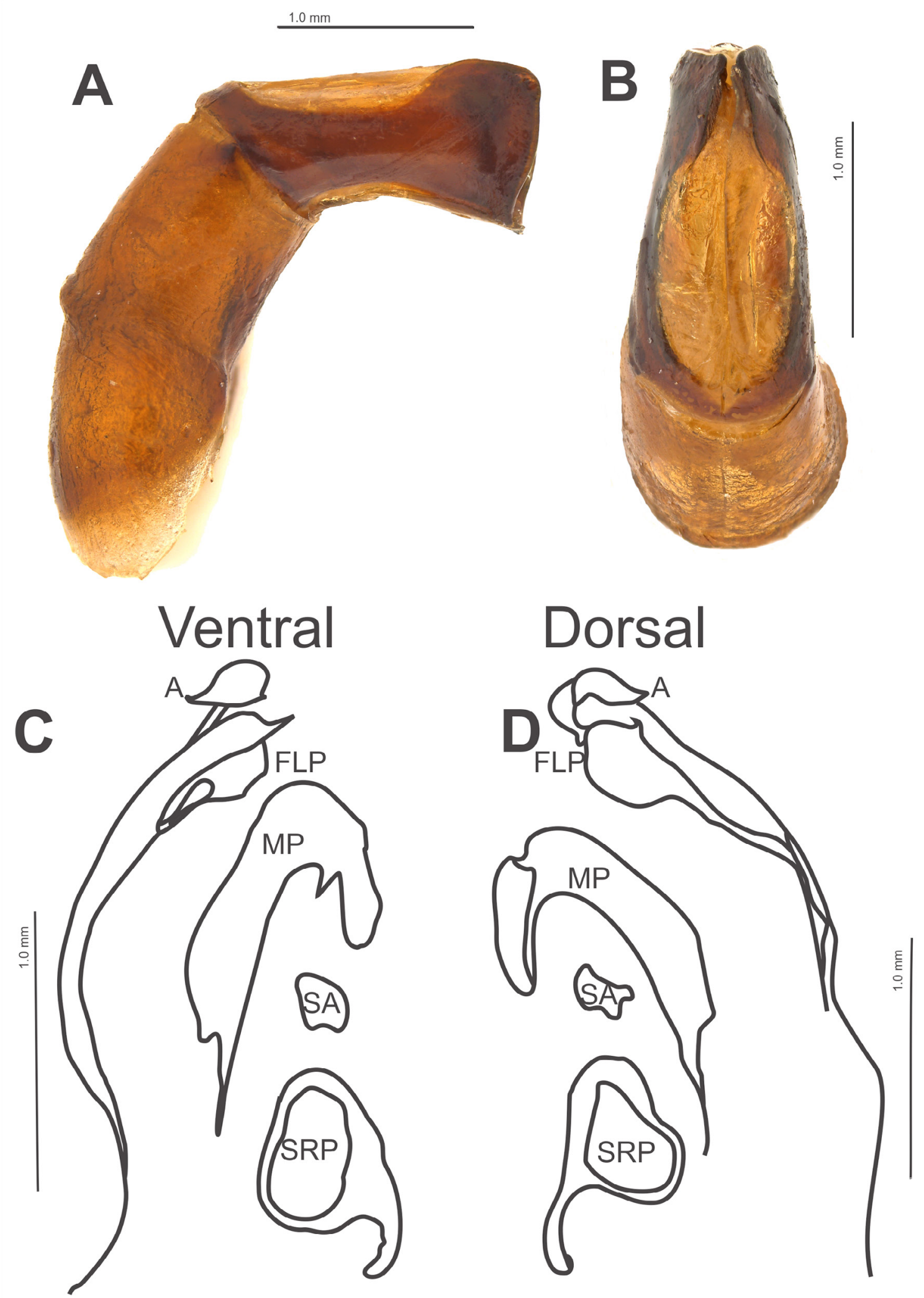

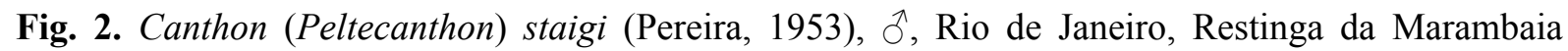
(CEMT). A. Parameres lateral view. B. Parameres dorsal view. C. Internal sac ventral view. D. Internal sac dorsal view. Abbreviations: see Material and methods. 
$45^{\circ} 25^{\prime 2} 24^{\prime \prime} \mathrm{W}$; CEMT • 2 q 9 ; same collection data as for preceding; $23^{\circ} 35^{\prime} 24^{\prime \prime} \mathrm{S}, 45^{\circ} 25^{\prime} 15^{\prime \prime} \mathrm{W}$; CEMT

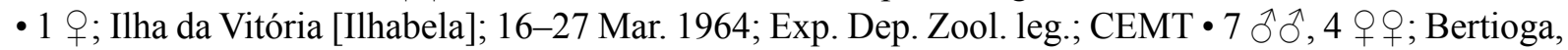
RPPN Sesc; $23^{\circ} 49^{\prime} 17^{\prime \prime}$ S, 46 $06^{\circ} 11^{\prime \prime}$ W; 16 m a.s.l.; Aug. 2014; R.V. Nunes leg.; restinga forest; pitfall hum. faec.; CEMT • 6 o $^{\wedge}$; Bertioga, RPPN Sesc; 2349'17" S, 4607'20" W; 16 Dec. 2014; R.V. Nunes leg.; gleba, carrion; CEMT • 1 spec.; São Vicente; Dec. 1935; CEAH. - Paraná • 1 ô; Maringá, Aeroporto de Maringá; 14 Dec. 2001; A. Bello leg.; CEMT • 1 đ̊; Paranaguá, ParNa do Superagui; 2527'37.7" S, 48 13'49.9" W; 22 Mar. 2014; C.M.Q. Costa leg.; pitfall, "fezes humana"; CEMT. - No data $\cdot 1$ spec.; CEAH.

\section{Remarks}

This species was described by Pereira (1953) in the same publication as the description of the subgenus, for which the author simultaneously designated it as the type species.

\section{Distribution}

Atlantic Forest, from Pernambuco to Paranaguá, southeast of the state of Paraná. In the literature there are records for Argentina and Paraguay (Pereira \& Martínez 1956; Martínez 1959; Halffter \& Martínez 1967), but we have not examined any specimens from these localities (Fig. 10).

\section{Canthon (Peltecanthon) haroldi nom. nov.}

Figs 3-4

Canthon prasinus Harold, 1867: 78.

Peltecanthon sulcatus - Pereira 1953: 399

Canthon prasinus - Harold 1868: 14, 77 (species list); 1869: 992 (catalogue); 1875: 59 (catalogue) Gillet 1911: 32 (catalogue) — Schmidt 1920: 125 (species list, first time as a synonym).

Canthon sulcatus [error] - Castelnau, 1840: 69 (catalogue) - Gillet 1911:34 (catalogue) - Schmidt 1920: 125 (species list); 1922: 69, 81 (key) — Balthasar 1939: 203 (key).

Canthon prasinum - Blackwelder 1944: 201 (catalogue).

Canthon sulcatum [error] - Blackwelder 1944: 202 (catalogue).

Peltecanthon sulcatus [error] - Vulcano \& Pereira 1964: 574 (catalogue) — Halffter \& Martínez 1967: 80 (monograph).

Canton (Peltecanthon) sulcatus [error] - Halffter \& Martínez 1977: 75.

Canton (Peltecanthon) sulcatum [error] - Vaz-de-Mello 2000: 191 (species list) - Cupello \& Vaz-deMello 2018: 46 - Vaz-de-Mello \& Cupello 2018a: 46.

\section{Diagnosis}

In the subgenus, Canthon haroldi can be separated by the following combination of characters. Elytra and pronotum dorsally green with shades of yellow and red (Fig. 3A). Metafemora with sulcus extending from the base and up to $1 / 3$ of the total femur length (Fig. 3B). Hypomere with no carina between the anterior and posterior portion (Fig. 3C). Propygidium with uniform line between pygidium and propygidium, without any central carina (Fig. 3D). Parameres flattened in lateral view (Fig. 4A); slightly sinuous apex never strongly sinuous or truncated, with a slight depression near the upper angle; rounded upper angle; lower angle slightly less than $90^{\circ}$. In dorsal view (Fig. 4B): membranous portion separates the parameres, wider in the central region and narrower at the base and apex. Internal sac (Fig. 4C-D) with the peripheral fronto-lateral sclerite (FLP) involving the axial sclerite (A), with the two structures forming a complex with a central duct, wider at the base, becoming abruptly tapered toward the apex; subaxial sclerite (SA) absent; peripheral medial sclerite (MP) lateral in relation to FLP + A, this with the sharper part passing within a semiduct formed by MP; MP with a central appendage at the inner margin (Fig. 4C); superior right peripheral sclerite (SRP) circular and with lateral prolongation. 


\section{Material examined}

Types

BRAZIL - 0 , lectotype of Canthon prasinus Harold, 1867, here designated; 1 (white label) "prasinus Har" / 2 (white label) "Bahia" / 3 (white label) "Ex-Museo E. Harold" / 4 (red label) "Type" / 5 (white label with black margins) "Peltecanthon sulcatus (Castelnau), det. G. Halffter, 67" / 6 (green label) "Museum París, ex Coll. R. Oberthur" / 7 (red label, black border) "LECTOTYPE Canthon prasinus Harold, des. F. Z. Vaz-de-Mello, 2014 (MNHN)".

\section{Other material (304 specs)}

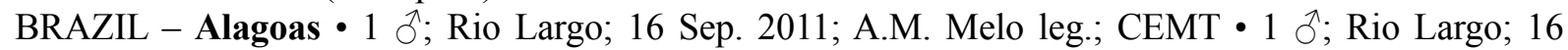
Mar. 2012; R.C. Nascimento leg.; CEMT • 1 O; Rio Largo; 15 Mar. 2013; M.B. Junior leg.; CEMT. Bahia • 1 '’; Nilo Peçanha, Mata do Jatimane; UTM24L0495129/8486655 [3902'42" W, 1341'21" S], 10 Feb. 2015; G.R.G. Sila et al. leg.; CEMT • 1 đ, 2 우; Costa do Sauípe; 14 Nov. 2004; I. Castro

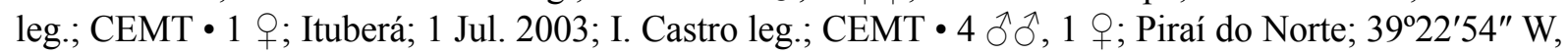

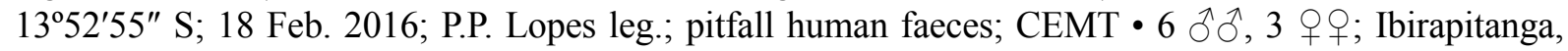
Mata do Ppuã; UTM24L0450355/8462777 [39²7'34" W, 1354'17" S]; 14 Nov. 2014; G.R.G. Sila et al. leg.; CEMT • 1 spec.; Rio Una; Oct. 1937; CEAH • 1 spec.; Rio Una; 23 Oct. 1937; Zellibor-Hauff leg.; CEAH • 1 q; Simões Filho, CIA Reserva; 17 Feb. 1987; D.H. Smith leg.; CEMT • 3 $\lesssim$ ô; Igrapiuna, Vila 5; 39¹0'13" W, 1348'8" S; 19 May 2010; R. Carvalho leg.; CEMT • 2 우여 Santa Cruz de Cabrália;

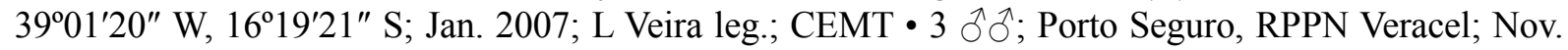
2004; J.C.N. Louzada leg.; pitfall human faeces; CEMT 1 ô; same collection data as for preceding; Feb. 2006; F.Z. Vaz-de-Mello leg.; pitfall human faeces; CEMT • 7 q ; Eunápolis, RPPN Veracel; Feb. 2006; F.Z. Vaz-de-Mello leg.; CEMT • 2 q ; Reserva do Maraú; 26 Jun. 2017; A.C. Amorim and Laboratório de Biodiversidade e Genética de Insetos leg.; CEMT. - Minas Gerais • 1 đ̊; Viçosa; 11 Jul. 1994; Almeida leg.; CEMT • 1 क; P.E. do Rio Doce; Oct. 1991; J.C.N. Louzada leg.; CEMT • 1 क; P.E. do Rio Doce; Feb. 1992; J.C.N. Louzada leg.; CEMT • 1 Oૈ, 2 우; P.E. do Rio Doce; 11 Oct. 1992; J. Louzada leg.; CEMT • 1 O; Marliéria, P.E. do Rio Doce; 14 Sep. 2001; S.S.P. Almeida leg.; CEMT • 1 §̊; Marliéria, P.E. do Rio Doce; 22 Sep. 2000; J.P. Soares leg.; CEMT • 1 ठ, 3 q $q$; Leopoldina, Recreio; Dec. 1999;

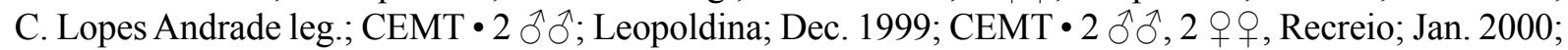
Lopes-Andrade and Vaz de Mello leg.; CEMT $\bullet 1 \delta^{\lambda}$; same collection data as for preceding; C. Lopes

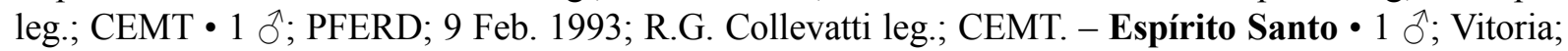
25 Mar. 1986; R.P. Moure leg.; CEMT • 1 \&; Jacaranema, B. Jucu; Apr. 1985; A.P. Aguiar leg.; restinga;

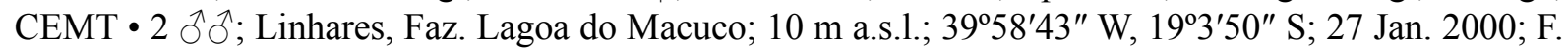
Génier and S. Ide leg.; prim. lowland Atlantic for., sandy ex. dung tp 4, day 1, FG2000-40; CEMT 1 ô, 1 우 Regência, Reserva Biológica de Comboios; 3953'5" W, 19³9'57" S; Jan. 2007; L. Vieira; CEMT - 1 q; Matataízes; Jan. 2007; L Vieira leg.; CEMT. - Espírito Santo, Linhares, Reserva Natural Vale

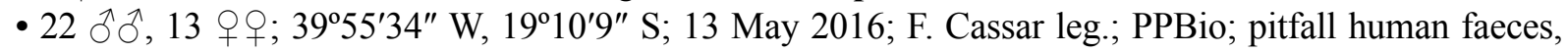

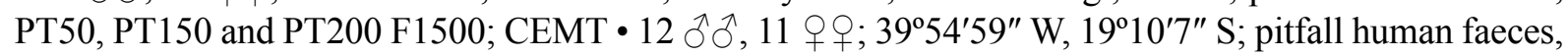

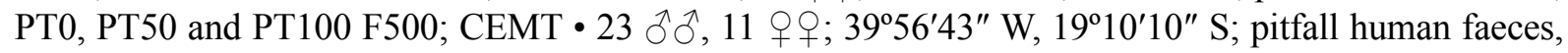
PT0, PT50 and PT150 F3500; CEMT • 12 $^{\prime} 0^{\lambda}, 8$ 우; 39 56 $6^{\prime} 8^{\prime \prime} \mathrm{W}, 19^{\circ} 10^{\prime} 10^{\prime \prime} \mathrm{S}$; pitfall human faeces,

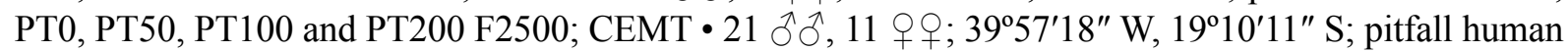

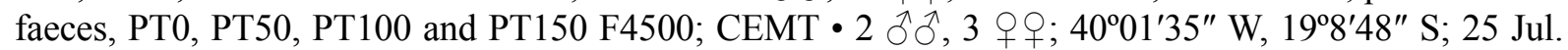

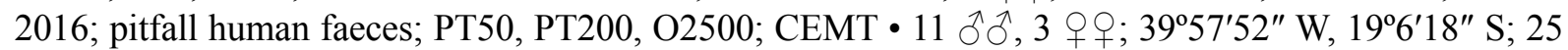

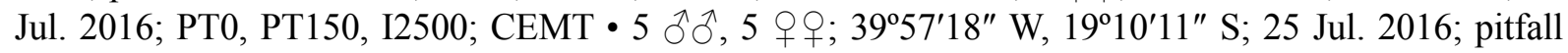

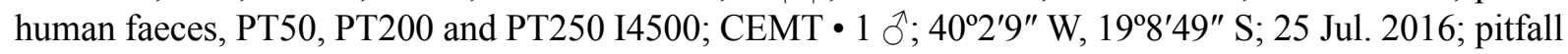

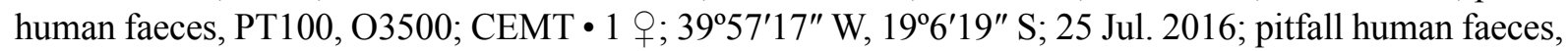

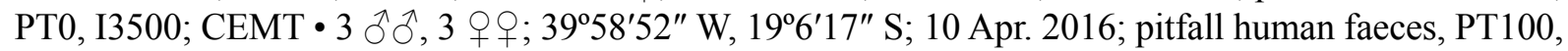
PT200, I500; CEMT • 1 क; 3958'27" W, 196'18"S; 10 Apr. 2016; pitfall human faeces, PT250, I1500; CEMT -3 우; same collection data as for preceding; pitfall human faeces, PT100, PT250, I1500; CEMT

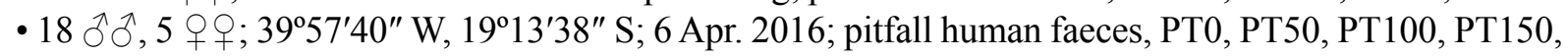



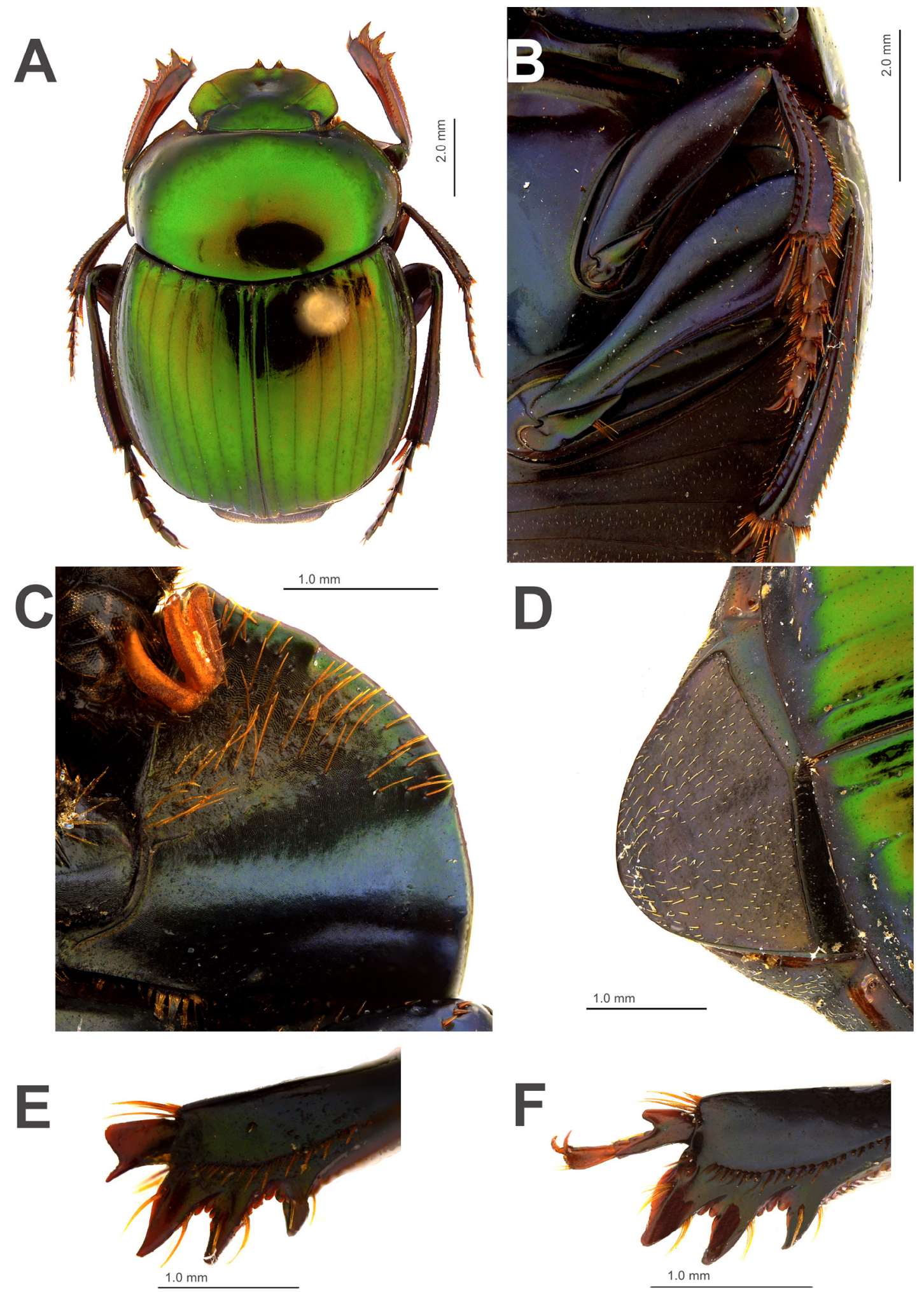

Fig. 3. Canthon (Peltecanthon) haroldi Harold, 1867 nom. nov. A-E. Male, Porto Seguro, RPPN Veracel (CEMT). A. Dorsal habitus. B. Ventral surface of meso- and metafemora. C. Hypomeron without transverse carina. D. Margin between pygidium and propygidium without central carina. E. Male protibial spur. F. Female protibial spur, Marliéria, P.E. do Rio Doce (CEMT). 

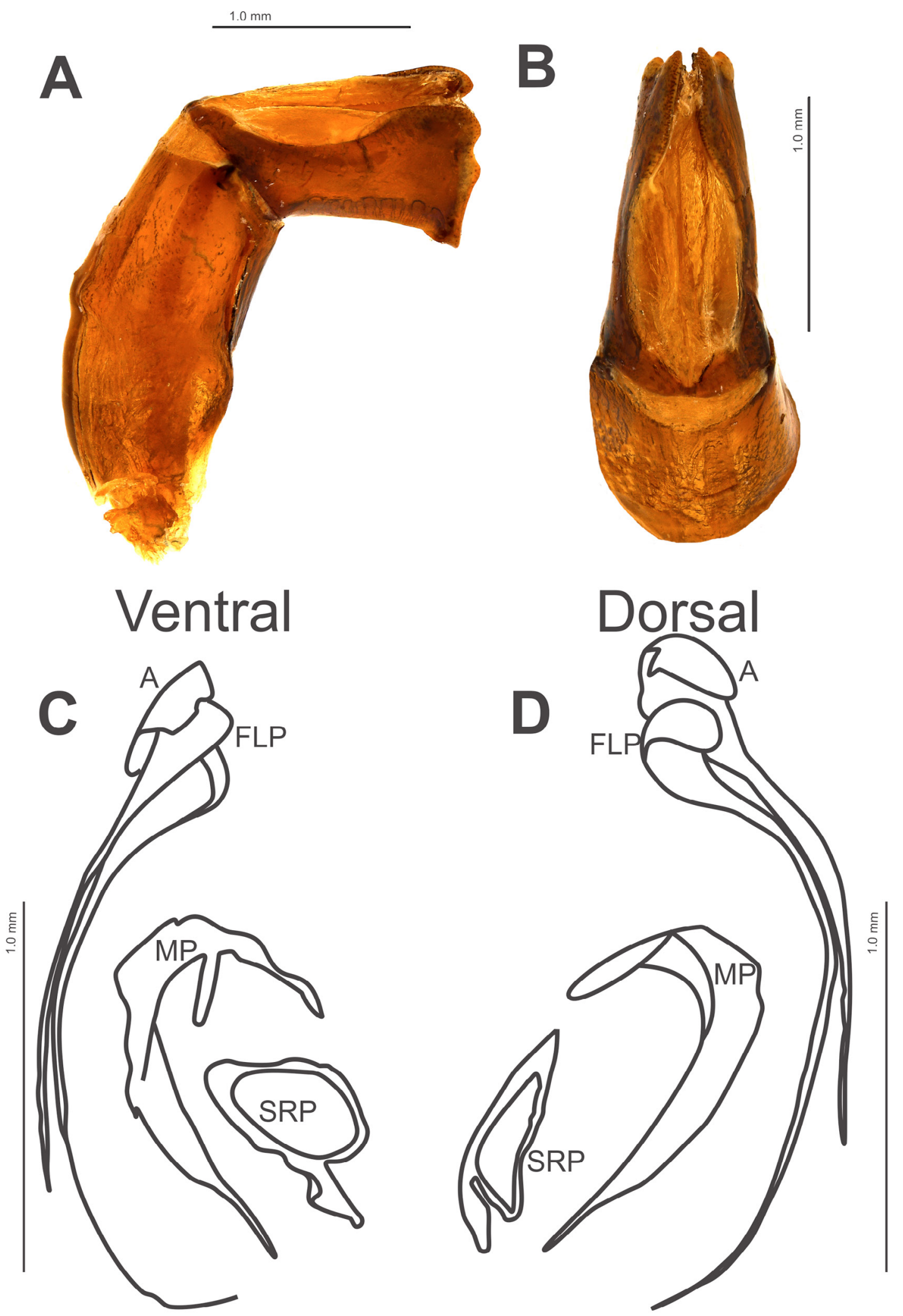

Fig. 4. Canthon (Peltecanthon) haroldi Harold, 1867 nom. nov., $\hat{\jmath}$, Bahia, Igrapiuna (CEMT). A. Parameres lateral view. B. Parameres dorsal view. C. Internal sac ventral view. D. Internal sac dorsal view. Abbreviations: see Material and methods. 


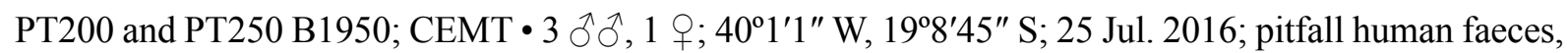

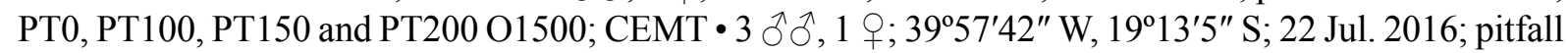

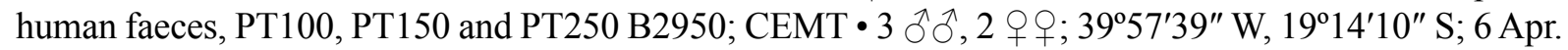

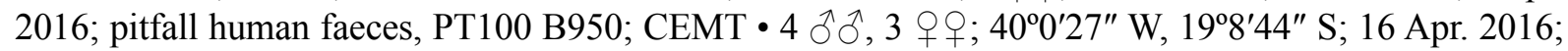

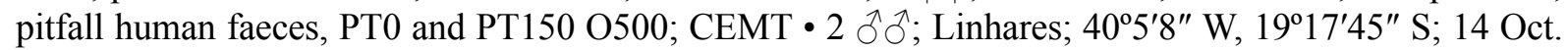
2012; R.C. Lima leg.; CEMT - Rio de Janeiro • 1 त̂; Campos dos Goytacazes, Fazenda Babilônia; 20 Apr. 2008; J.S. Multani leg.; CEMT・1 $\overbrace{}^{\top}$; no collecting data; CEMT.

\section{Remarks}

This species has a complex and confusing nomenclatural history. The name Canthon prasinus was first established by Klug (1833) for a new species from Madagascar. However, Klug's species was not described in a suitable genus since Canthon is entirely Neotropical in occurrence. Four years later, Dejean (1836) transferred that species to his new genus Epilissus, of which Reiche (1841) subsequently designated it as the type species.

More than three decades after Reiche's work, Harold (1867a) established a primary junior homonym C. prasinus for a Brazilian species that, according to Harold (1868), had been misidentified as Canthon sulcatus Perty, 1830 by Castelnau (1840); Perty's actual species, in fact, belonged to Canthidium Erichson, 1847, where it had already been transferred by Harold (1867b) and still remains (Cupello 2018). In his 1869 catalogue of the world Scarabaeinae, Harold (1869: 992) maintained that stance by including 'C. sulcatus Castelnau' as a junior synonym of $C$. prasinus Harold. Harold (1875) used $C$. prasinus as valid again in his list of the dung beetles of the municipality of Cantagalo, Rio de Janeiro state, Brazil.

Gillet (1911) listed Canthon prasinus Harold and 'Canthon sulcatus Castelnau' as valid species; however, in the same work, he also considered the two names as synonyms.

In most of the 'recent' literature, only one author followed Gillet's view (Blackwelder 1944), while many others followed Schmidt's (Balthasar 1939; Pereira 1953; Vulcano \& Pereira 1964), but all those authors were wrong. This included the descriptive work of the new South American genus Peltecanthon (Pereira 1953).

Applying Article 23.9.5 of the International Code of Zoological Nomenclature, there are arguments that both names can be preserved, since they have not been considered congeneric after 1899. However, to invoke that Article, we would have to consider that $C$. prasinus is currently in use, which is not the case, or that $C$. sulcatus was first published by Schmidt, not Castelnau (which is also not the case). Considering this context, we propose Canthon haroldi as a new replacement name, in homage to Edgar Von Harold, who made the most correct evaluation regarding the application of those names, and was misinterpreted by subsequent authors.

\section{Distribution}

Atlantic Forest in the Brazilian coast, in the states of Bahia, Espírito Santo, Minas Gerais and Rio de Janeiro (Fig. 10).

Canthon (Peltecanthon) splendidus Schmidt, 1922

Figs 5-6

Canthon splendidus Schmidt, 1922: 68, 81, 93.

Canthon splendidus - Balthasar 1939: 209 (key) — Halffter \& Martínez 1977: 76 (as junior synonym of Canthon auricollis).

Canthon (Peltecanthon) splendidus - Vaz-de-Mello \& Cupello 2018a: 46; 2018b: 67-68, figs 101-102. 


\section{Diagnosis}

Canthon splendidus can be distinguished by the following combination of characters: elytra and pronotum with different colouration, with pronotum bright with golden or red reflections, and elytra blue-green (Fig. 5A). In the legs the metafemora with no groove at base (Fig. 5B). Hypomere with a strong excavation in the anterior region, with a thick transverse carina between the anterior and posterior portion projecting to near the lateral margin and never only up to the median region (Fig. 5C). Propygidium with a moderate carina near pygidium border (Fig. 5D). In lateral view In lateral view (Fig. 6A) parameres flattened; a strongly sinuous apex, never slightly sinuous or truncated, with a deep excavation near the upper angle; rounded upper angle; lower angle much less than $90^{\circ}$. In dorsal view (Fig. 6B): membranous portion separates the parameres, wider in the central region and narrower at the base and apex. Internal sac (Fig. 6C-D) with peripheral fronto-lateral sclerite (FLP) involving the axial sclerite (A), where the two structures form a complex with a central duct, wider at the base, with a subtle tapering in the median region and sharp apex; subaxial sclerite (SA) absent; peripheral medial sclerite (MP) lateral to FLP + A, tapered region with the sharper part passing within a semiduct formed by MP; superior right peripheral sclerite (SRP) circular and with lateral prolongation.

\section{Material examined}

\section{Lectotype}

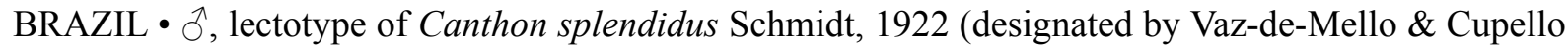
2018b: 68); 1 (white label) "Canthon splendidus A. Schmidt n. sp." / 2 (green label) "Esp. Santo" / 3 (green label) "Coll C Felsche, Kauf 20, 1918" / 4 (red label, black border) "LECTOTYPE Canthon splendidus Schmidt, des. F. Z. Vaz-de-Mello, 2014 (SMTD)”.

\section{Paralectotypes}

BRAZIL: 1 O’; 1 (green label) "Esp. Santo" / 2 (green label) "Coll C Felsche, Kauf 20, 1918”/ 3 (red label) "splendidus" / 4 (yellow label, black border) "PARALECTOTYPE Canthon splendidus Schmidt, des.

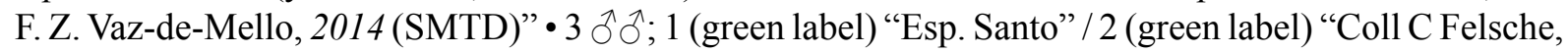
Kauf 20, 1918" / 3 (yellow label, black border) "PARALECTOTYPE Canthon splendidus Schmidt, des. F. Z. Vaz-de-Mello, 2014 (SMTD)".

Other material (18 specs)

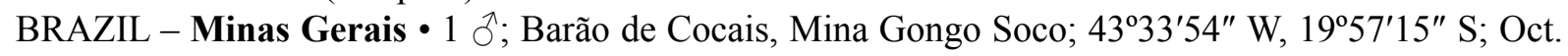

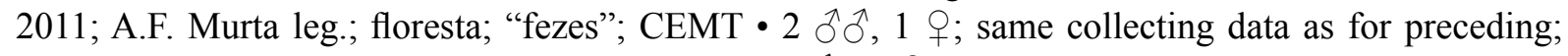

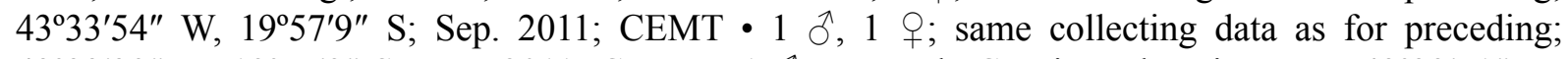

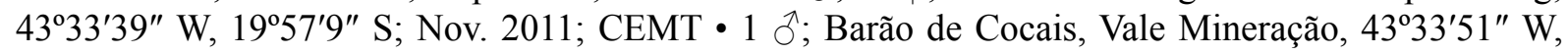
19 57'17" S; 860 m a.s.1.; 4 Nov. 2011; R.N. Mota leg.; forest, rain season; human dung; CEMT • 1 ; ; same collecting data as for preceding; $43^{\circ} 40^{\prime} 16^{\prime \prime} \mathrm{W}, 20^{\circ} 03^{\prime} 9^{\prime \prime} \mathrm{S} ; 1481 \mathrm{~m}$ a.s.1.; 10 Oct. 2010; R.N. Mota leg.; forest/rock field, dry season; human dung; CEMT • $3 \overbrace{}^{\lambda} \partial^{\lambda}$; Mariana, Mina Timbopeba;

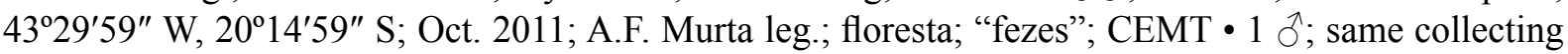

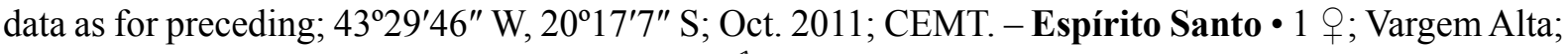
15 Sep. 1995; J.C.N. Louzada leg.; CEMT • 1 đ; same collecting data as for preceding; Louzada and Louzada leg.; mata, F\#4; CEMT 1 ภ, 1 क; same collecting data as for preceding; $680 \mathrm{~m}$ a.s.1.; J.C.N.

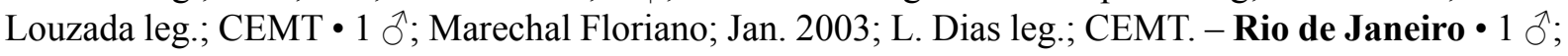
Itatiaia, PARNA de Itatiaia; $44^{\circ} 38^{\prime} 10^{\prime \prime} \mathrm{W}, 22^{\circ} 25^{\prime} 20^{\prime \prime} \mathrm{S} ; 1650 \mathrm{~m}$ a.s.1.; 23-26 Dec. 2011; C. Araújo and R. Andrade leg.; CEMT.

\section{Redescription}

Male

MeAsurements. Body length (except the head): $9.5 \mathrm{~mm}$, pronotum width: $6.0 \mathrm{~mm}$. 
CoLour. Dorsally, pronotum and elytra with distinct colouration elytra dark blue, pronotum green with bright red and yellow reflections (Fig. 5A).

HEAD. Green with bright yellow. Dorsal surface with small spots equally spaced. Two clypeal teeth triangular, with rounded apex, separated by a V-shaped emargination, with two dorsal tufts of setae and two ventral tufts. Clypeo-genal suture present (Fig. 5A).

Pronotum. Pronotal disc smooth, green with small bright red and yellow spots, equally spaced; Anterior angles acute (Fig. 5A).

ELYTRA. Striae narrow, always distinct; interstriae wide, flat.

HYPOMERON. Dark blue and black, chagrinated microsculpture; sparse setae present on anterior portion and anterolaterally; strong transverse carina along $2 / 3$ of the whole length; anterior portion little excavated, with prominent teeth anterolaterally (Fig. 5C).

LEGS. Protibiae with three teeth; protibial spur flattened dorsoventrally, bifid, with medial and lateral bifurcation, triangular, different sizes, asymmetrical (Fig. 5E). Meso- and metafemora with ventral side lacking anterior and posterior margin; metafemora with a narrower and longer base, claviform; metafemoral ventral surface with spots at the base (Fig. 5B).

Aвdomen. Ventrites with few setae, chagrinated, black and blue.

Pygidium. As wide as long; blue with bright green and yellow; basal margin little expanded (Fig. 5D).

Parameres. Flattened laterally; on lateral view, apical portion with a long sinuosity; on dorsal view, central membranous portion enlarged in the centre (Fig. 6A-B).

INTERNAL SAC. Fronto-lateral peripheral sclerite (FLP) involving axial sclerite (A), forming a central duct with spiniform apex; subaxial sclerite (SA) absent; medial peripheral sclerite (MP) semicircular, widely emarginated, with the internal margin with a lobe on the extremity (without central tooth); external margin sinuous with a lobe on the extremity, lobes separated by a U-shaped margin; apex of FLP + A involved by MP; superior right peripheral sclerite (SRP) circular with lateral prolongation in the dorsoventral direction (Fig. 6C-D).

Morphological VARiation. Body length (except the head) ranges from $9.5 \mathrm{~mm}$ to $12 \mathrm{~mm}$; pronotum width from 6 to $7.5 \mathrm{~mm}$. Pronotum with bright reflections, according to the viewing angle, between different intensities of green, red and yellow.

\section{Female}

LEGS. Protibial spur with medial and lateral bifurcation, triangular, equal in size, symmetrical (Fig. 5F).

\section{Remarks}

For a long time, this species was identified as Canthon auricollis Redtenbacher, 1868, but the examination of the type specimens of both nominal species allowed Vaz-de-Mello \& Cupello (2018a) to conclude that, in fact, the latter name refers to a another species belonging to Canthon s. str., closely related to C. lividus Blanchard, 1846. It is an insect that can be considered very rare and can only be collected at altitudes usually over $1000 \mathrm{~m}$. The predominance of pronotal sheen varies from golden yellow to red.

\section{Distribution}

High altitude in the Atlantic Forest along the coast of Brazil, in the states of Minas Gerais, Espírito Santo and Rio de Janeiro (Fig. 10). 


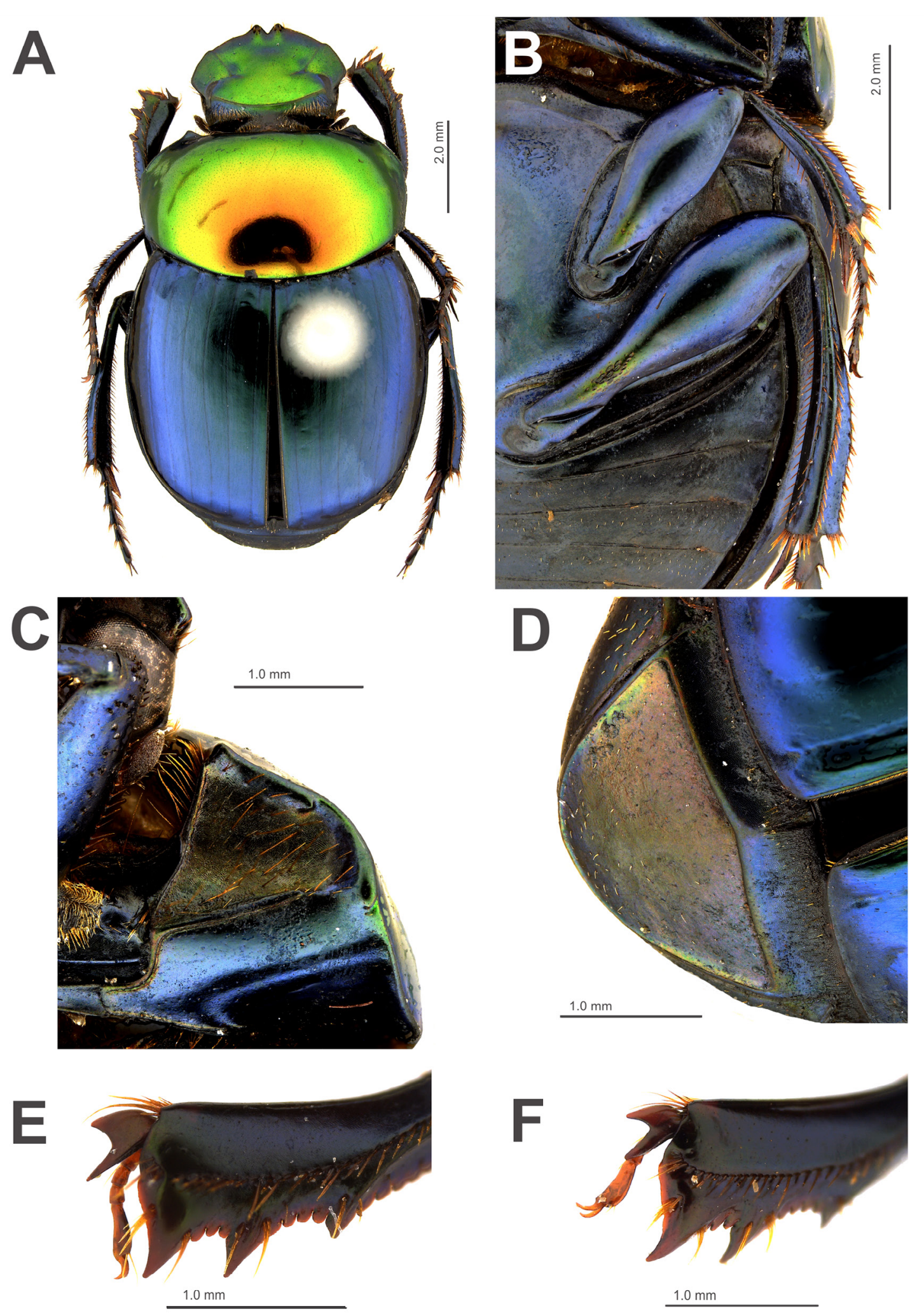

Fig. 5. Canthon (Peltecanthon) splendidus Schmidt, 1922. A-E. Male, Minas Gerais, Barão do Cocais, Mina Gongo Soco (CEMT). A. Dorsal habitus. B. Ventral surface of meso- and metafemora. C. Transverse carina in the hypomeron. D. Carina in the margin between pygidium and propygidium. E. Male protibial spur. F. Female protibial spur, Espírito Santo, Vargem Alta (CEMT). 

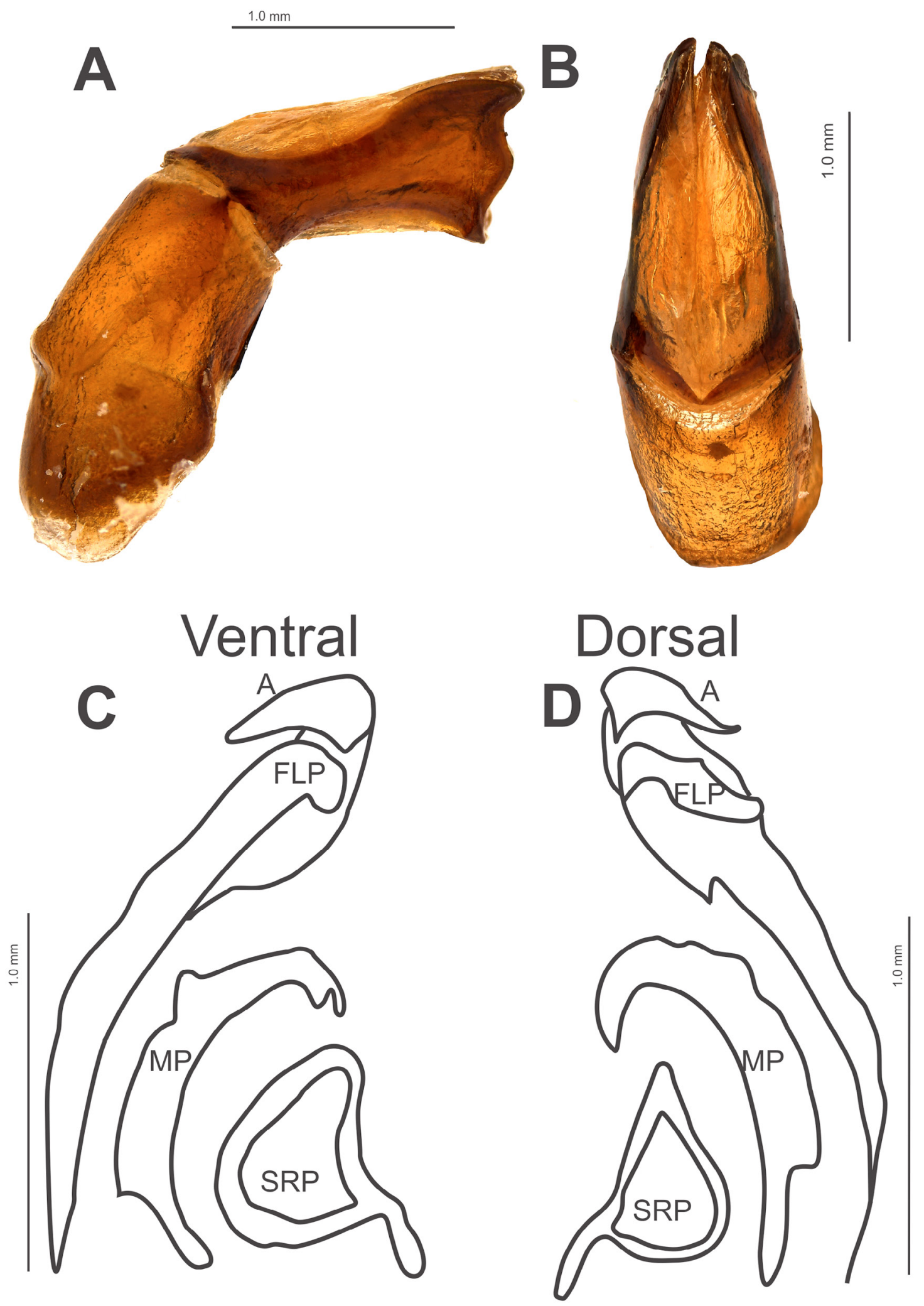

Fig. 6. Canthon (Peltecanthon) splendidus Schmidt, 1922, Ô, Rio de Janeiro, Marechal Floriano (CEMT). A. Parameres lateral view. B. Parameres dorsal view. C. Internal sac ventral view. D. Internal sac dorsal view. Abbreviations: see Material and methods. 


\section{Canthon (Peltecanthon) terciae sp. nov. urn:1sid:zoobank.org:act:C9F36543-7F9D-4498-ABAF-3784D46A2E60}

Figs 7-9

\section{Diagnosis}

Canthon terciae sp. nov. can be distinguished by the following combination of characters: elytra and pronotum dorsally red or green reddish (Fig. 7A). Legs with flat metafemur at the base, with no punctures or groove (Fig. 7B). Hypomeron with thin transverse carina between the anterior and posterior portion, projecting to the median region, never near the lateral margin, anterior region without excavation (Fig. 7C). Propygidium with strong carina near pygidium edge (Fig. 7D). Parameres, in lateral view (Fig. 8A): flattened; truncated apex, sub-straight, never sinuous; slightly rounded upper angle; lower right angle. In dorsal view (Fig. 8B): membranous portion separating the parameres, wider in the central region and narrower at the base and at the apex. Internal sac (Fig. 8C-D). Peripheral fronto-lateral sclerite (FLP) involving the axial sclerite (A), the two structures form a complex with a central duct, wider at the base, which gradually tapers to the apex, which is much thinner; subaxial sclerite (SA), smaller than in C. staigi, lateral to the FLP + A complex; peripheral medial sclerite (MP), without central dentin at the inner margin, distal to A, lateral in relation to FLP + A tapered region, with the sharper part passing within a semiduct formed by MP; superior right peripheral sclerite (SRP) with lateral angular prolongation.

\section{Etymology}

This species is named in honour of Dr Tercia Vargas dos Santos, who developed phylogeographical studies with the subgenus Peltecanthon and reinforced our taxonomic decision.

\section{Material examined}

\section{Holotype}

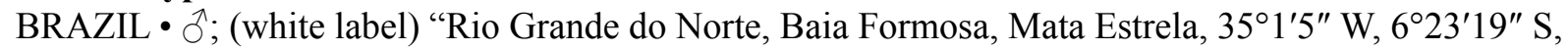
44 m, 24-V-2007, leg. J. A. Rafael \& F. F. Xavier F (CEMT)”.

\section{Paratypes (98 specs)}

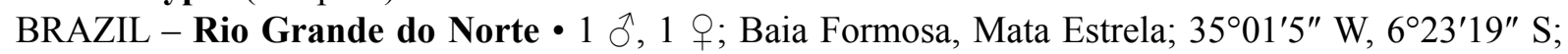

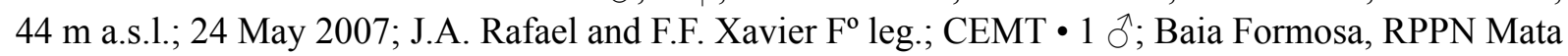
Estrela; 3501'0.7" W, 6022'53.2" S; 1 Oct. 2013; C.M.Q. Costa Endres leg.; pitfall, "fezes humanas";

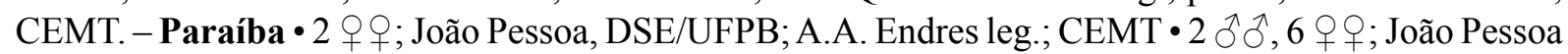
[Lagoa Seca]; 35 $50^{\prime}$ W, $7^{\circ} 10^{\prime}$ S; May 2002; M.I.M. Hernández leg.; "floresta"; "armadilha pitfall";

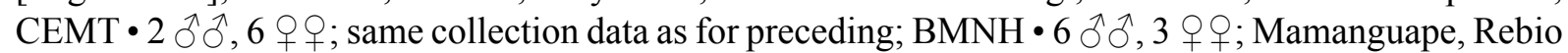
Guaribas; 35 $15^{\prime}$ W, 641' S; 2002; M.I.M. Hernández leg.; "floresta"; "armadilha de interceptação de

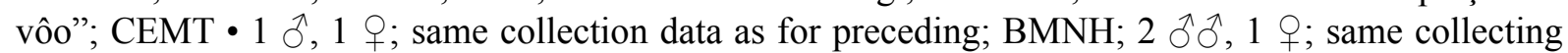
data as for preceding; Jan.-Feb. and Apr. 2002; A.A. Endres leg.; "mata"; "armadilha pitfall"; CEMT

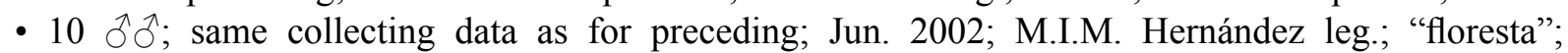

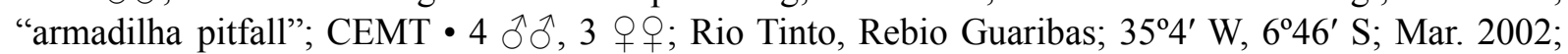
M.I.M. Hernández leg.; "floresta"; "armadilha pitfall"; CEMT • 1 đ̊; Mamanguape, Rebio Guaribas; 35¹1'58.8" W, 643'32.3" S; 30 Sep. 2013; C.M.Q. Costa Endres leg.; pitfall, "fezes humanas"; CEMT • 2 9 ㅇ Guaribas, Reserva Biológica de Guaribas; 3 May 2014; Laboratório de Biodiversidade e Genética

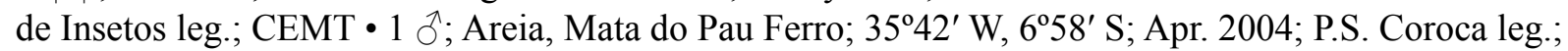

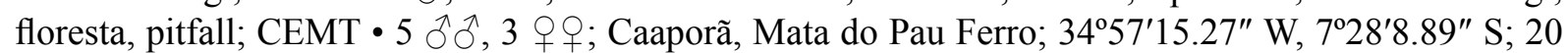
Apr. 2013; C.N. Liberal leg.; frag. A, pitfall 3; "fezes"; CEMT • 1 §, 1 क; same collection data as for preceding; BMNH. - Pernambuco - 3 q ; Itamaracá, Vila Velha; 11-13 May 2011; Laboratório de Biodiversidade e Genética de Insetos leg.; CEMT $\bullet 1$ \%; same collection data as for preceding; 5 Oct. 

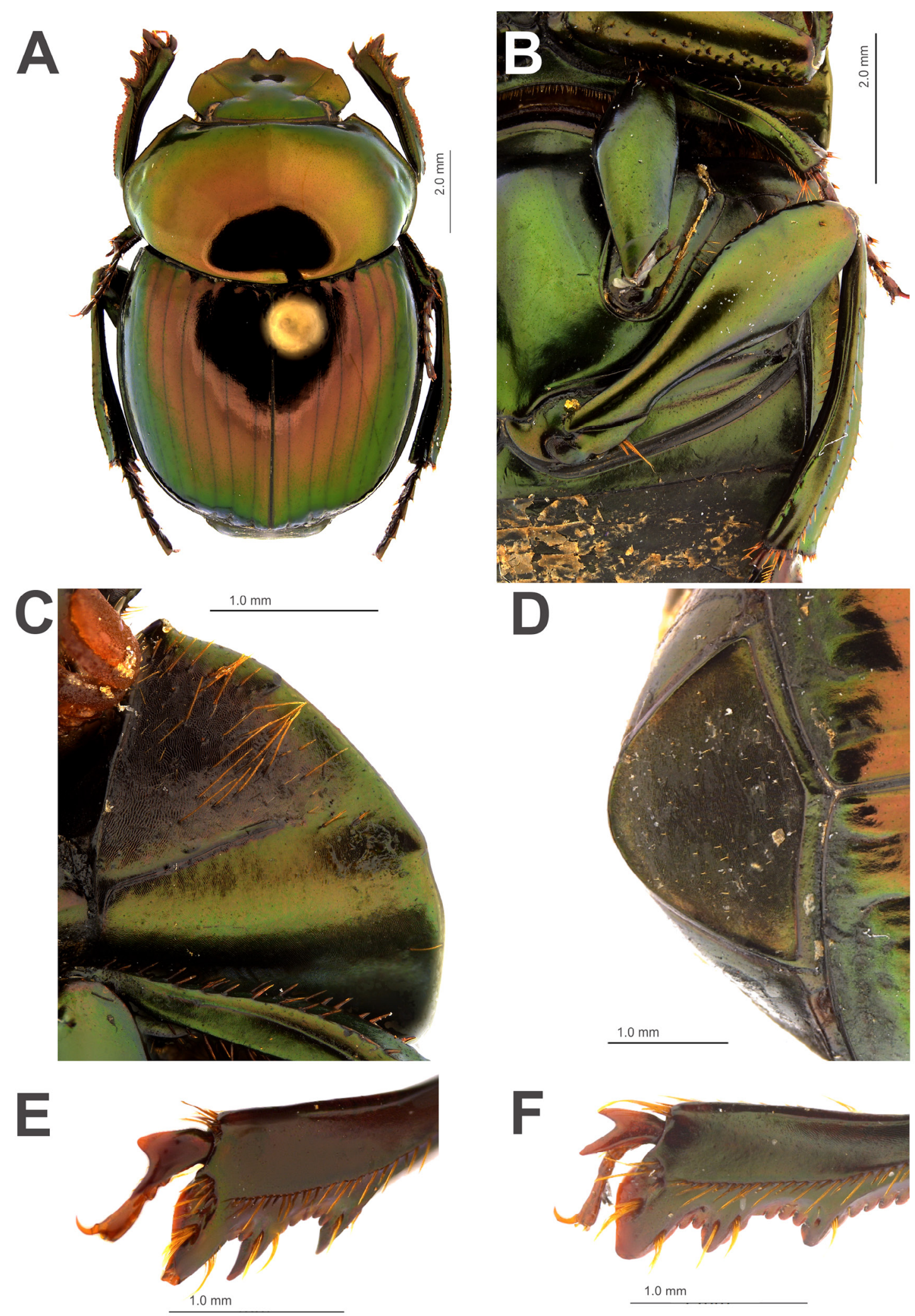

Fig. 7. Canthon (Peltecanthon) terciae sp. nov., paratype, $\widehat{\partial}$, Rio Grande do Norte, Baia Formosa, Mata Estrela (CEMT). A. Dorsal habitus. B. Ventral surface of meso- and metafemora. C. Transverse carina in the hypomeron. D. Carina in the margin between pygidium and propygidium. E. Male protibial spur. F. Paratype,, , Rio Grande do Norte, Baia Formosa, Mata Estrela (CEMT), protibial spur. 
2011; CEMT • 2 $\widehat{\jmath}$; Araçoiaba, CIMNC; 17 May 2010; K.K.T. Pessoa leg.; pitfall (carne), fechada 4, conjunto 2; CEMT $\bullet 1$ ऽ, 1 q; same collecting data as for preceding; 28 Feb. 2010; K.K.T. Pessoa

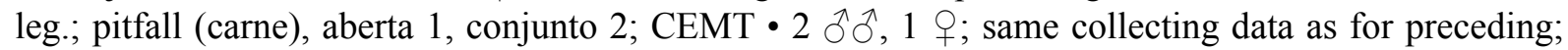
15 May 2010; R.S. Correia leg.; (P. A.) carne e fezes, fechada 1 and 2 - 17:00; CEMT $\bullet 3$ ôे; same collecting data as for preceding; 17 Apr. 2010; R.S. Correia leg.; (P. A.) fezes e carne, fechada 1 and

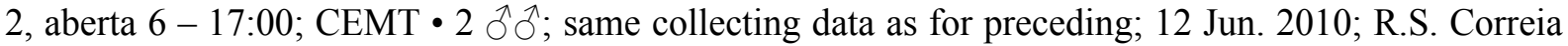

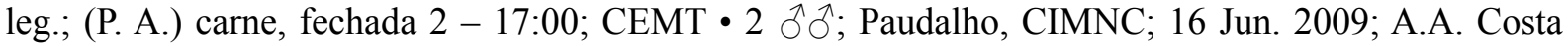

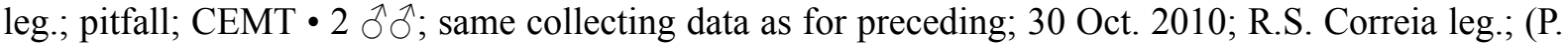
A.) carne, fechada $1-17: 00 ;$ CEMT $\bullet 1 \hat{0}, 2$ + $O$; same collecting data as for preceding; 31 Oct. 2010; R.S. Correia leg.; (P. A.) carne, fechada 1 - 7:00; CEMT • 3 q $\circ$; Caruaru; 5 Jul. 2012; Laboratório de Biodiversidade e Genética de Insetos leg.; CEMT • 1 ภ, 3 우; Pesqueira, Serra do Ororubá [Ororoba]; 1 Jul. 2013; Laboratório de Biodiversidade e Genética de Insetos leg.; CEMT • 1 đ , 2 우; Bonito, Brejo

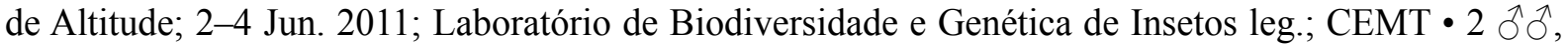
1 q; Tamandaré; 8 Jan. 2010; R.C. Moura and Laboratório de Biodiversidade e Genética de Insetos leg.; CEMT. - Sergipe - 2 क 9 ; Santa Luzia do Itanhy; 26 Jun. 2017; I.C. Amorin and Laboratório de Biodiversidade e Genética de Insetos leg.; CEMT.

\section{Description}

\section{Holotype (male)}

MEASUREMENTS. Body length (except the head): $10.0 \mathrm{~mm}$, pronotum width: $7.0 \mathrm{~mm}$.

CoLour. Dorsally red with bright green. Ventrally green with bright red (Fig. 7A).

HEAD. Red with bright green. Dorsal surface with small spots equally spaced. Two clypeal teeth triangular, with rounded apex, separated by a V-shaped emargination, with two tuft of setae dorsally and two tufts ventrally. Clypeo-genal suture present (Fig. 7A).

Pronotum. Pronotal disc smooth, red, bright, with small punctures equally spaced. Anterior angles acute (Fig. 7A).

ELYTRA. Striae narrow, distinctly impressed. Interstriae wide, flat (Fig. 7A).

Hypomeron. Green, chagrinated microsculpture. Sparse setae present on anterior portion and anterolaterally. With thin transverse carina between the anterior and posterior portion projecting to the median, region anterior not excavated (Fig. 7C). Anterior portion flat, with a little tooth anterolaterally (Fig. 7C).

Legs. Protibiae with three teeth. Protibial spur flattened dorsoventrally, bifid, with medial and lateral triangular bifurcation with different sizes, asymmetrical (Fig. 7E). Meso- and metafemoral ventral side lacking anterior and posterior margin. Metafemora with a narrower and longer base, claviform (Fig. 7B).

ABDOMEN. Ventrite glabrous, chagrinated. Black and green.

Pygidium. As wide as long. Red with bright green. Basal margin expanded, forming a strong carina in V-form between pygidium and propygidium.

Parameres. Flattened laterally. On lateral view, apical portion ventrally truncated, dorsally a little curve. In dorsal view, central membranous portion enlarged in the centre (Fig. 8A-B).

INTERNAL SAC (Figs 8-9). Fronto-lateral peripheric sclerite (FLP) involving axial sclerite (A) forming a central duct with spiniform apex, very slim. Subaxial sclerite (SA) small, lateral to FLP + A complex and 

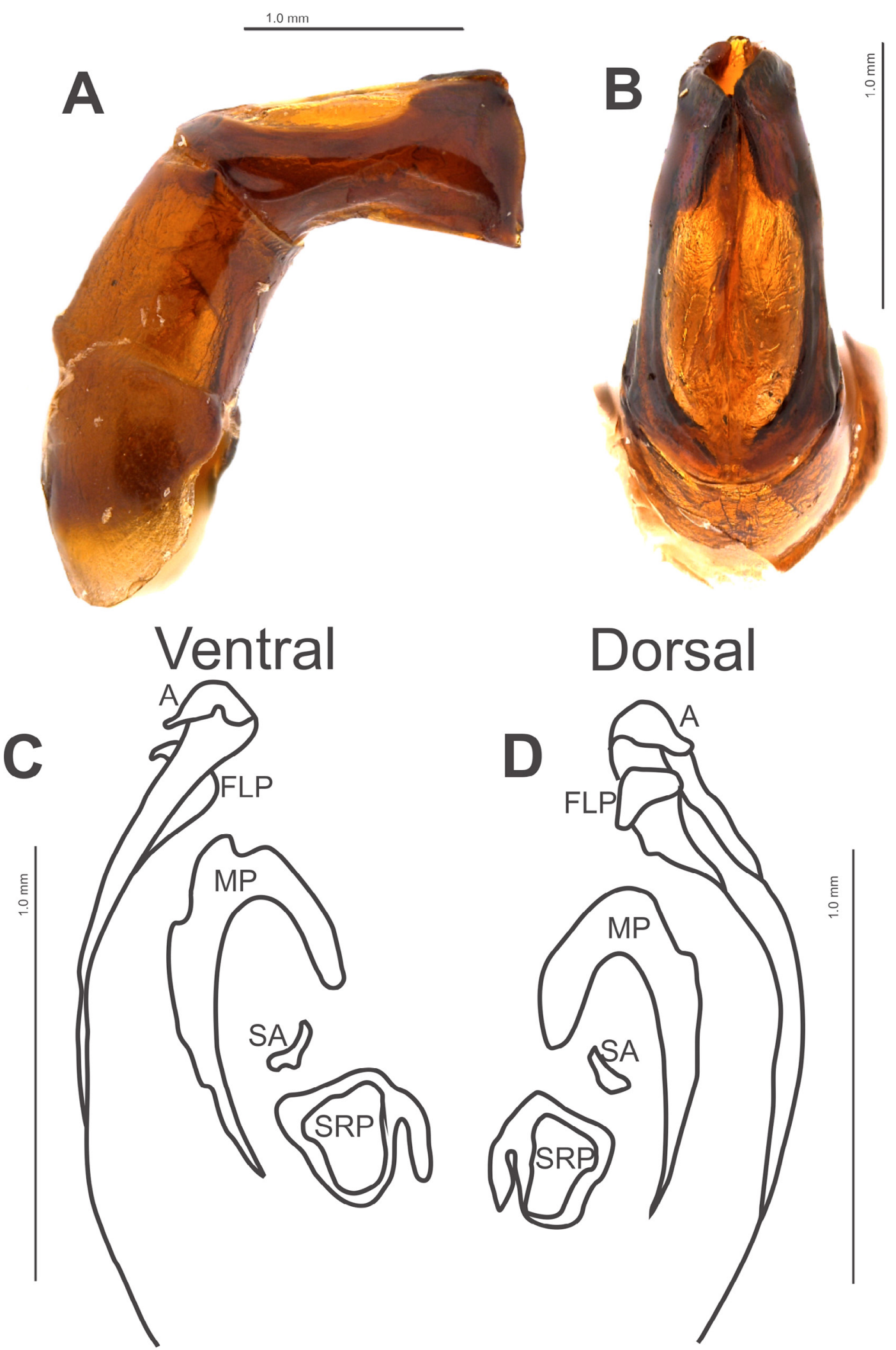

Fig. 8. Canthon (Peltecanthon) terciae sp. nov., paratype, $\widehat{\partial}$, Rio Grande do Norte, Baia Formosa, Mata Estrela (CEMT). A. Parameres lateral view. B. Parameres dorsal view. C. Internal sac ventral view. D. Internal sac dorsal view. Abbreviations: see Material and methods. 

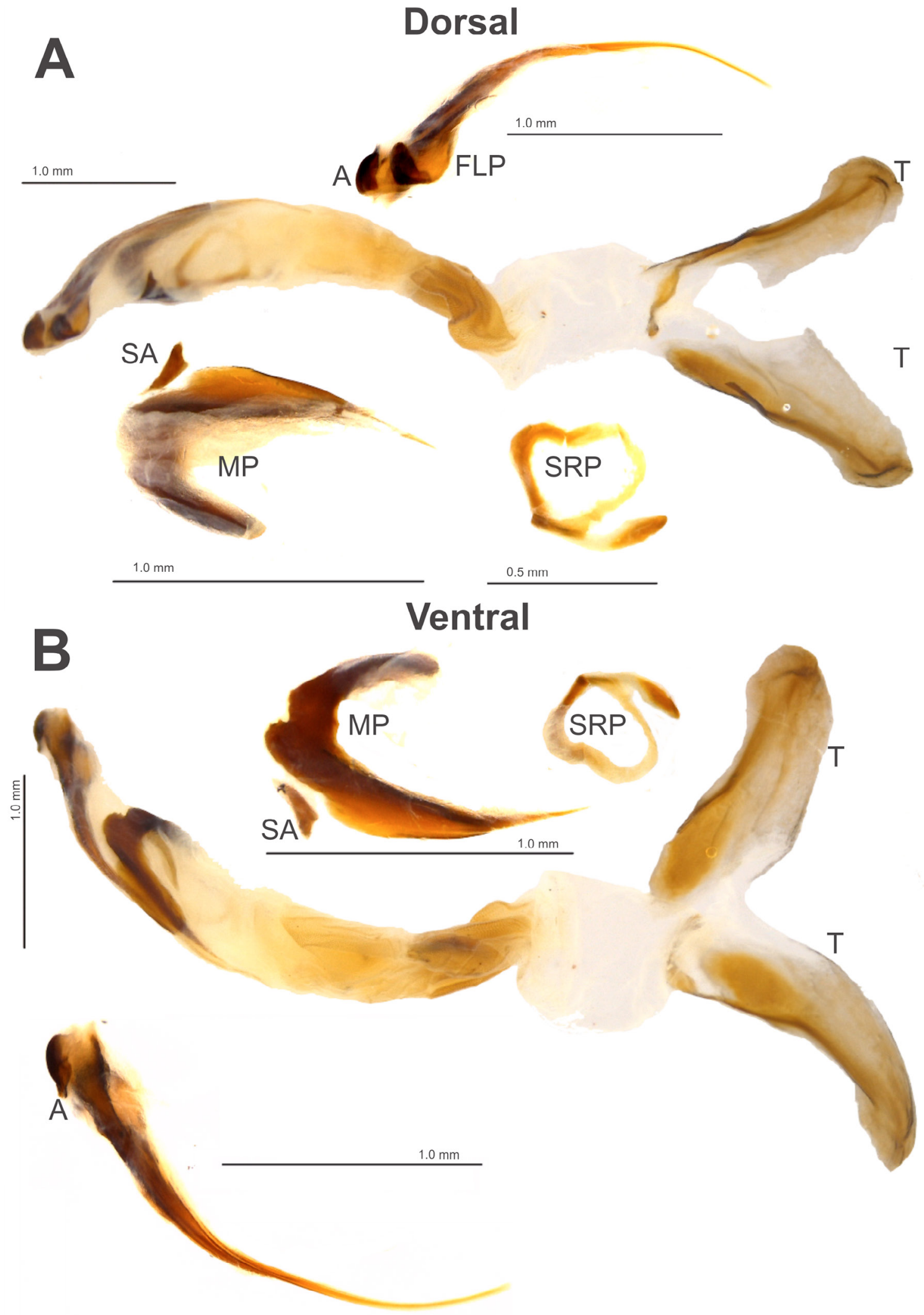

Fig. 9. Canthon (Peltecanthon) terciae sp. nov., paratype, $\widehat{\partial}$, Rio Grande do Norte, Baia Formosa, Mata Estrela (CEMT). Position of the internal sac sclerites. A. Dorsal view. B. Ventral view. Abbreviations: see Material and methods. 
MP, dorsal to all sclerites. Medial peripheric sclerite (MP) semicircular, widely emarginated, with the internal margin continue (without central tooth), margin external sinuous; Apex of the FLP + A complex involved by MP. Superior right peripheral sclerite (SRP) circular, with lateral prolongation in the dorsoventral direction.

Morphological VARIATION. Body length (except the head) ranges from 8.5 to $11.5 \mathrm{~mm}$; pronotum width from 6 to $7.5 \mathrm{~mm}$. Colour: dorsally green with bright red. Legs: metafemoral ventral surface not completely smooth, with some punctures.

\section{Female}

LEGS. Protibial spur with medial and lateral bifurcation triangular with equal sizes, symmetrical (Fig. 7F).

\section{Remarks}

This new species is much more similar to C. staigi than to the other two species of Peltecanthon. The differences between them are very subtle, and there are great morphological similarities between them, in the main characters that differentiate the species of this subgenus; the main differences observed are in the sclerites of the internal sac (exactly in MP). In addition to the sclerite, these species (C. staigi and $C$. terciae sp. nov.) can be differentiated only by the characters of the dorsal colour and base of the metafemur, without any need for dissection.

\section{Geographic distribution}

Northern Brazilian Atlantic Forest, in the states of Rio Grande do Norte, Paraíba, Pernambuco and Sergipe (Fig. 10).

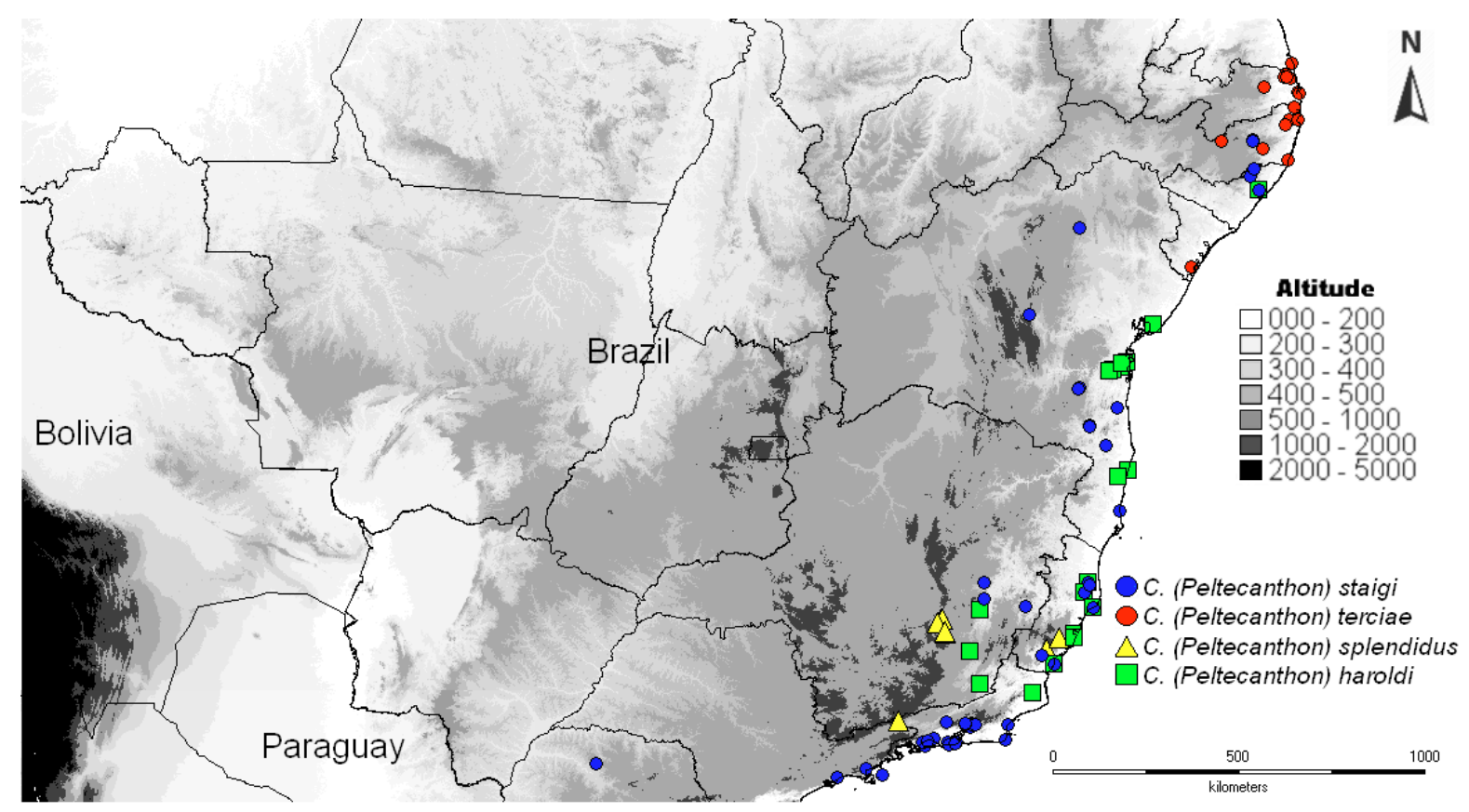

Fig. 10. Distribution map for species of Peltecanthon: C. (P.) staigi (Pereira, 1953) (blue circle); C. (P.) haroldi Harold, 1867 nom. nov. (green square); C. (P.) splendidus Schmidt, 1922 (yellow triangle); $C$. (P.) terciae sp. nov. (red circle) 


\section{Discussion}

Phylogenetic relationships between the groups within Canthon are still poorly understood. Despite the fact that Medina et al. (2003) placed Peltecanthon near Goniocanthon, their analysis does not consider molecular data and lacks some important species. However, there is a close external resemblance between the two subgenera (Peltecanthon and Goniocanthon), but we cannot conclude at this point whether this resemblance is due to morphological convergence or common ancestry.

However, despite their colour and dorsal resemblance, one can readily distinguish the two genera by looking at the pygidium (flat in Peltecanthon, strongly convex in Goniocanthon) and metafemora (claviform in Peltecanthon, regular in Goniocanthon) (Nunes et al. 2018). Both characters are very useful in avoiding misidentification, especially between $C$. (Peltecanthon) staigi and C. (Goniocanthon) smaragdulus (Fabricius, 1781), both blue-coloured species from the Brazilian Atlantic Forest.

At first glance, Peltecanthon species diversity is related to historically stable areas in the Atlantic Forest, as defined by Carnaval \& Moritz (2008). Recent published data on Scarabaeinae (genus Dichotomius Hope, 1837) also reveal that the northern part of the Atlantic Forest harbours several endemic species (Valois et al.2017). Extra attention is needed to remnants of Atlantic forest: first, because of a considerable number of new endemic species awaiting description around Alagoas, Pernambuco and Sergipe forest remnants. Second, these remnants are in great danger, mainly from anthropogenic activities (Zwiener et al. 2017).

Little information is available on the ecology and behaviour of Peltecanthon from labels and from the published literature. In general, all species seem to be attracted to mammalian faeces and carrion. The occurrence of $C$. staigi does not seem to depend on well-preserved habitats, and it is sometimes the 'last' Scarabaeinae species in heavily disturbed environments (pers. obs. RVN). Of the other species, $C$. splendidus is the rarest, $C$. terciae sp. nov. appears to be quite common in parts of the northeast coast and C. haroldi is very abundant in the region of Linhares in Espírito Santo.

Further descriptive taxonomic work on Canthon and other Deltochilini groups is required. It is imperative to develop morphological and molecular phylogenies to provide better classifications, and a better understanding of Neotropical Scarabaeinae systematics and evolution. However, alphataxonomy description works are still essential to elucidate, at least, the diversity of the Atlantic Forest Scarabaeinae.

\section{Acknowledgments}

LGOAN (23108.096582/2015-11) and RVN (23108.036500/13-3) received CAPES grants. FZVM receives financial support from CNPq (process $n^{\circ}$ 302997/2013-0, 484035/2013-4, 440604/2015-0, 568005/2014 PRONEM, 248299/2012-3, 405697/2013-9), Synthesis (GB-TAF-3855), INCT-CENBAM/ $\mathrm{CNPq}$ and Rede ComCerrado/MMA. We thank all curators named in the methods section, and Mario Cupello for providing geographical records.

\section{References}

Balthasar V. 1939. Eine Vorstudie zur Monographie der Gattung Canthon Hffsg. Folia Zoologica et Hydrobiologica 9 (2): 179-238.

Blackwelder R. 1944. Checklist of the Coleopterous insects of Mexico, Central America the West Indies and South America. Part 2. Bulletin of the United States National Museum 185: 189-341.

https://doi.org/10.5479/si.03629236.185.2 
Carnaval A.C. \& Moritz C. 2008. Historical climate modelling predicts patterns of current biodiversity in the Brazilian Atlantic forest. Journal of Biogeography 35: 1187-1201.

https://doi.org/10.1111/j.1365-2699.2007.01870.x

Castelnau Comte de. 1840. Histoire Naturelle des Insectes Coléoptères. Tome Deuxième. Société bibliophile, Paris. https://doi.org/10.5962/bhl.title.36538

Costa Lima A. da 1953. Insetos do Brasil; 8. ${ }^{\circ}$ Tomo Capitulo XXIX, Coleópteros 2. ${ }^{a}$ parte. Escola Nacional de Agronomia Série Didática N. ${ }^{\circ} 10$. Rio de Janeiro.

Cupello M. 2018. On the type species of the New World dung beetle genus Canthidium Erichson, 1847 (Coleoptera: Scarabaeidae: Scarabaeinae), with an annotated checklist of species. Zootaxa 4388 (4): 451-486. https://doi.org/10.11646/zootaxa.4388.4.1

Cupello M. \& Vaz-de-Mello F.Z. 2018. A monographic revision of the Neotropical dung beetle genus Sylvicanthon Halffter \& Martínez, 1977 (Coleoptera: Scarabaeidae: Scarabaeinae: Deltochilini), including a reappraisal of the taxonomic history of 'Canthon sensu lato'. European Journal of Taxonomy 467: 1-205. https://doi.org/10.5852/ejt.2018.467

Dejean P.F.M.A. 1836. [livraison 1-4, pp. 1-384]. In: Dejean P.F.M.A. 1836-1837. Catalogue des Coléoptères de la Collection de M. le Comte Dejean. Troisième édition, revue, corrigée et augmentée Méquignon-Marvis Père et Fils, Paris [publication date according to Madge, 1988].

Ferreira-Neto C.A., Cruz G.A. dos S., Amorim I.C., Balbino V.Q. \& Moura R. de C. 2017. Effects of fragmentation and anthropic pressure on the genetic structure of Canthon (Peltecanthon) staigi (Coleoptera: Scarabaeidae) populations in the Atlantic Forest domain. Journal of Insect Conservation 21 (2): 267-276. https://doi.org/10.1007/s10841-017-9980-4

Harold E. 1869. Catalogus Coleopterorum Hucusque Descriptorum Synonymicus et Systematicus. Tome IV: Scarabaeidae. E.H. Gummi, Munich. https://doi.org/10.5962/bhl.title.9089

Gillet J.J.E. 1911. Coleopterorum Catalogus. Pars 38. Scarabaeidae: Coprinae I. W. Junk, Berlin.

Halffter G. 1961. Monografía de las especies norteamericanas del género Canthon. Ciencia, Revista Hispano-Americana de Ciencias Puras y Aplicadas 20 (9-12): 225-320.

Halffter G. \& Martínez A. 1967. Revisión monográfica de los Canthonina Americanos. Revista de la sociedade mexicana de historia natural 28 (2): 79-117.

Halffter G. \& Martínez A. 1977. Revisión monográfica de los Canthonina Americanos IV parte. Clave para géneros y subgéneros. Folia entomologica mexicana 38: 29-107.

Harold E. 1867a. Diagnosen neuer Coprophagen. Coleopterologische Hefte 1: 76-83. Available from https://biodiversitylibrary.org/page/16069331 [accessed 16 Dec. 2019].

Harold E. 1867b. Zur Kenntniss der Gattung Canthidium und ihrer nächsten Verwandten. Coleopterologische Hefte 1: 1-61. Available from https://biodiversitylibrary.org/page/16069237 [accessed 29 Dec. 2018].

Harold E. 1868. Monographie der Gattung Canthon. Berliner Entomologische Zeitschrift 12: 1-144. Available from https://biodiversitylibrary.org/page/8344235 [accessed 16 Dec. 2019].

Harold E. 1875. Verzeichniss der von Dr. Teuscher in Cantagallo gesammelten coprophagen Lamellicornien. Coleopterologische Hefte 13: 57-72.

Klug J.C. 1833. Bericht über eine auf Madagascar veranstaltete Sammlung von Insecten aus der Ordnung Coleoptera. Eine in der Königl. Akademie der Wissenschaften am 29. März 1832 gelesene Abhandlung. Mit fünf illuminirien Tafeln. Königlichen Akademie der Wissenschaften, Berlin. 
Madge R.B. 1988. The publication dates of Dejean's catalogues. Archives of Natural History 15: $317-$ 321. https://doi.org/10.3366/anh.1988.15.3.317

Martínez A. 1959. Catalogue de los Scarabaeidae Argentinos. Revista del Museo Argentino de Ciencias Naturales “Bernardino Rivadavia”, Ciencias Zoológicas 5 (1): 1-126.

Medina C.A., Scholtz C.H. \& Gill B.D. 2003. Morphological variation and systematics of Canthon Hoffmansegg 1817, and related genera of new world Canthonini dung beetles (Coleoptera, Scarabaeinae). Deutsche Entomologische Zeitschrift 50 (1): 23-68. https://doi.org/10.1002/mmnd.20030500105

Nunes L.G.O.A., Nunes R.V. \& Vaz-de-Mello F.Z. 2018. Taxonomic revision of the South American subgenus Canthon (Goniocanthon) Pereira \& Martínez, 1956 (Coleoptera: Scarabaeidae: Scarabaeinae: Deltochilini). European Journal of Taxonomy 437: 1-31. https://doi.org/10.5852/ejt.2018.437

Pereira F.S. 1953. Notas sinonímicas (Col. Scarabaeidae). Dusenia 4 (5-6): 387-402.

Pereira F.S. \& Martínez A. 1956. Os gêneros de canthonini americanos (Col. Scarabaeidae). Revista Brasileira de Entomologia 6: 91-152.

Reiche L. 1841. Tableau d'une division systématique de la tribu des Coprophages, dans la famille des Lamellicornes. Revue et magasin de zoologie, pure et appliquée 4: 211-213.

Schmidt A. 1920. Beitrag zur kenntnis der Gattungen Canthon Hffsg., Sybax Boh, Aphodius Ill, Simogenius Har., Ataenius Har. Archiv für Naturgeschichte A 86 (9): 114-147.

Schmidt A. 1922, 1. Bestimmungstabelle der mir bekannten Canthon-Arten. 2. Verbreitungsbiete der Canthon-Arten. 3. Neubeschreibungen von Canthon, Saprosites, Mendidius, Euparia und Ataenius. Archiv für Naturgeschichte 88 (3): 61-103.

Tarasov S.I. \& Dimitrov D. 2016. Multigene phylogenetic analysis redefines dung beetles relationships and classification (Coleoptera: Scarabaeidae: Scarabaeinae). BioMed Central Evolutionary Biology 16: 257. https://doi.org/10.1186/s12862-016-0822-x

Tarasov S.I. \& Génier F. 2015. Innovative Bayesian and parsimony phylogeny of dung beetles (Coleoptera, Scarabaeidae, Scarabaeinae) enhanced by ontology-based partitioning of morphological characters. PLoS ONE 10 (3): 1-86. https://doi.org/10.1371/journal.pone.0116671

Tarasov S.I. \& Solodovnikov A.Y. 2011. Phylogenetic analysis reveal reliable morphological markers to classify mega-diversity in Onthophagini dung beetles (Coleoptera: Scarabaeidae: Scarabaeinae). Cladistics 27: 1-39. https://doi.org/10.1111/j.1096-0031.2011.00351.x

Valois M., Vaz-de-Mello F.Z. \& Silva F.A.B. 2017. Taxonomic revision of the Dichotomius sericeus (Harold, 1867) species group (Coleoptera: Scarabaeidae: Scarabaeinae). Zootaxa 4277 (4): 503530. https://doi.org/10.11646/zootaxa.4277.4.3

Vaz-de-Mello F.Z. 2000. Estado atual de conhecimentos dos Scarabaeidae s. str. (Coleoptera: Scarabaeoidea) do Brasil. In: Martín-Piera F., Morrone J.J. \& Melic A. (eds) Hacia un Proyecto CYTED para el Inventario y Estimación de la Diversidad Entomológica en Iberoamérica: PRIBES-2000, Vol. 1: 183-195. Monografías Tercer Milenio y Sociedad Entomológica Aragonesa, Zaragoza.

Vaz-de-Mello F.Z. \& Cupello M. 2018a. The type specimens of South American dung beetles, Part II: The species described by the Austrian coleopterist Ludwig Redtenbacher (1814-1876) in the 1868 part of the "Reise der österreichischen Fregatte Novara um die Erde" (Insecta: Coleoptera: Scarabaeidae: Scarabaeinae). Annalen des Naturhistorischen Museums in Wien, B 120: $41-58$. 
Vaz-de-Mello F.Z. \& Cupello M. 2018b. The type specimens of South American dung beetles. Part I: On the species described in the genus Canthon Hoffmannsegg, 1817 by the German entomologist Adolf Schmidt (1856-1923) (Coleoptera, Scarabaeidae, Scarabaeinae). Spixiana 41 (1): 33-76.

Vaz-de-Mello F.Z., Edmonds W.D., Ocampo F. \& Schoolmeesters P. 2011. A multilingual key to the genera and subgenera of the subfamily Scarabaeinae of the New World (Coleoptera: Scarabaeidae). Zootaxa 2854: 1-73. https://doi.org/10.11646/zootaxa.2854.1.1

Vulcano M.A. \& Pereira F.S. 1964. Catalogue of the Canthonini (Col. Scarab.) inhabiting the Western Hemisphere. Entomologischen Arbeiten aus dem Museum G. Frey 15: 570-685.

Zwiener V.P., Padial A.A., Marques M.C.M., Faleiro F.V., Loyola R. \& Townsend P.A. 2017. Planning for conservation and restoration under climate and land use change in the Brazilian Atlantic Forest. Diversity and Distributions 1-12. https://doi.org/10.1111/ddi.12588

Zunino M. 1978. L'armatura genitale negli Onthophagini: tecniche di preparazione e criteri di studio. L'informatore del giovane entomologo. Bollettino della Società Entomologica Italiana, Suppl. 90: 2126.

Manuscript received: 2 January 2019

Manuscript accepted: 4 October 2019

Published on: 17 January 2020

Topic editor: Gavin Broad

Section editor: Max Barclay

Desk editor: Pepe Fernández

Printed versions of all papers are also deposited in the libraries of the institutes that are members of the EJT consortium: Muséum national d'histoire naturelle, Paris, France; Meise Botanic Garden, Belgium; Royal Museum for Central Africa, Tervuren, Belgium; Royal Belgian Institute of Natural Sciences, Brussels, Belgium; Natural History Museum of Denmark, Copenhagen, Denmark; Naturalis Biodiversity Center, Leiden, the Netherlands; Museo Nacional de Ciencias Naturales-CSIC, Madrid, Spain; Real Jardín Botánico de Madrid CSIC, Spain; Zoological Research Museum Alexander Koenig, Bonn, Germany; National Museum, Prague, Czech Republic. 